\title{
GLOBAL OSCILLATORY WAVES FOR SECOND ORDER QUASILINEAR WAVE EQUATIONS
}

\author{
PAUL GODIN
}

\begin{abstract}
In this paper we prove the global existence and describe the asymptotic behaviour of a family of oscillatory solutions of Cauchy problems for a class of scalar second order quasilinear wave equations, when the space dimension is odd and at least equal to 3 . If time is bounded, corresponding results for quasilinear first order systems were obtained by Guès; to prove our results we reduce our problems to bounded time problems with the help of a conformal inversion. To obtain global results, suitable geometric assumptions must be made on the set where the oscillations are concentrated at initial time.
\end{abstract}

\section{INTRODUCTION}

In recent years much work has been done on the existence, for small or bounded time, of oscillatory solutions of nonlinear first order hyperbolic systems (see e.g. [5, 6], and the references given there). The purpose of the present paper is to prove the global existence and describe the asymptotic behaviour of a family of oscillatory solutions of Cauchy problems for a class of scalar second order quasilinear wave equations when the space dimension is odd and at least equal to 3 . Global existence results of smooth solutions of second order quasilinear wave equations with small initial data have been obtained in $[8,9$, $1,10]$. The class of equations which will be considered here appears in [1], but, in the present paper, the initial data will have high frequency oscillations and hence will not, in general, satisfy the smallness conditions of $[1,8,9,10]$. To prove our results, we shall reduce our problems to bounded time problems by means of a conformal transformation, in the spirit of [1] (see also [4])).

Our paper is organized as follows. In $\S 2$, we state our results precisely. In $\S 3$ we recall some useful properties of the Kelvin transform $([13,4])$ which will be used to reduce our global problems to local ones. Finally Theorems 1 and 2 are proved in $\S \S 4$ and 5 respectively.

Received by the editors October 19, 1993.

1991 Mathematics Subject Classification. Primary 35L70, 35C20, 35B40.

Key words and phrases. Quasilinear second order wave equations, global existence, oscillatory solutions, conformal inversion. 


\section{StATEMENT OF THE Results}

We consider the Cauchy problem

$$
\begin{gathered}
\square z=\sum_{0 \leq i, j \leq N} f^{i j}\left(z, z^{\prime}\right) \partial_{i j}^{2} z+f\left(z, z^{\prime}\right) \quad \text { if } t>t_{0}, x \in \mathbb{R}^{N}, \\
\partial_{0}^{k} z=\bar{z}_{k} \quad \text { if } t=t_{0}, x \in \mathbb{R}^{N}, k=0,1 .
\end{gathered}
$$

Here $x=\left(x_{1}, \ldots, x_{N}\right)$ is the space variable, $t$ is the time variable, $t_{0} \in$ $\mathbb{R}$ is fixed, $\partial_{0}=\partial / \partial t, \partial_{i}=\partial / \partial x_{i}$ if $1 \leq i \leq N, \square=\partial_{0}^{2}-\sum_{1 \leq i \leq N} \partial_{i}^{2}$, $z^{\prime}=\left(\partial_{0} z, \partial_{1} z, \ldots, \partial_{N} z\right)$, and $\partial_{i j}^{2}=\partial_{i} \partial_{j} . f^{i j}$ and $f$ are $C^{\infty}$ in an open neighbourhood of $(0,0)$ in $\mathbb{R} \times \mathbb{R}^{N+1}, f^{i j}=f^{j i}$ for all $i, j$, and $f^{i j}(0,0)=$ $\partial^{\alpha} f^{i j}(0,0)=0$ if $|\alpha| \leq 1$, so that the right-hand side of $(2.1)$ is at least quadratic. We assume that $\bar{z}_{k} \in C_{0}^{\infty}\left(\mathbb{R}^{N}\right)$ if $k=0,1$. (The reason for not supposing that $t_{0}=0$ in problem (2.1), (2.2) will be explained in Remark 2.6 below.) In order to prove global results, we shall assume that $N$ is odd and $\geq 3$, and if $N=3$, that the null condition of $[9,1]$ is satisfied, namely

(i) $f^{i j}(z, p)=b \eta^{i j} z+\sum_{0 \leq k \leq 3} b^{i j k} p_{k}+\mathscr{O}\left(z^{2}+|p|^{2}\right)$ as $(z, p) \rightarrow(0,0)$ in $\mathbb{R} \times \mathbb{R}^{4}$, where $p=\left(p_{0}, p_{1}, p_{2}, p_{3}\right), \eta^{00}=1, \eta^{i i}=-1$ if $i>0, \eta^{i j}=$ 0 if $i \neq j, b \in \mathbb{R}, b^{i j k} \in \mathbb{R}$, and $\sum_{0 \leq i, j, k \leq 3} b^{i j k} q_{i} q_{j} q_{k}=0$ for all $q=$ $\left(q_{0}, q_{1}, q_{2}, q_{3}\right) \in \mathbb{R}^{4}$ which satisfy $q_{0}^{2}=\sum_{1 \leq k \leq 3} q_{k}^{2}$;

(ii) $f(z, p)=\sum_{0 \leq i, j \leq 3} b^{i j} p_{i} p_{j}+\mathscr{O}\left(|z|^{3}+|p|^{3}\right)$ as $(z, p) \rightarrow(0,0)$ in $\mathbb{R} \times \mathbb{R}^{4}$, where $b^{i j} \in \mathbb{R}$ are such that $\sum_{0 \leq i, j \leq 3} b^{i j} q_{i} q_{j}=0$ for all $q=\left(q_{0}, q_{1}, q_{2}, q_{3}\right) \in$ $\mathbb{R}^{4}$ which satisfy $q_{0}^{2}=\sum_{1 \leq k \leq 3} q_{k}^{2}$.

Under the preceding assumptions, when the $C_{0}^{\infty}\left(\mathbb{R}^{N}\right)$ functions $\bar{z}_{k}, k=$ 0,1 , have small Sobolev $H^{(N+5) / 2-k}$ norm, it follows from the results of [1] that $(2.1),(2.2)$ has a global $C^{\infty}$ solution (see also $\left.[8,9,10]\right)$. In the present paper we are going to consider a class of oscillatory initial data $\bar{z}_{k} \in C_{0}^{\infty}\left(\mathbb{R}^{N}\right)$ which are not necessarily small in $H^{(N+5) / 2-k}$ (but only in $C^{1-k}$ ). Now we describe the structure of these Cauchy data. Assume that $\psi \in C^{\infty}\left(\mathbb{R}^{N}\right)$ has the following property: there exist $a, b \in \mathbb{R}$ with $a<b$ such that, for each $\lambda \in[a, b]$, (i) $\psi^{\prime}(x) \neq 0$ if $\psi(x)=\lambda$ and (ii) $\Omega_{\lambda}=\left\{x \in \mathbb{R}^{N}, \psi(x)<\lambda\right\}$ is a bounded strongly convex open set (by strongly convex we mean that $\partial \Omega_{\lambda}$ is connected and that the following holds: if $x \in \partial \Omega_{\lambda}$ and $v \in \mathbb{R}^{N} \backslash\{0\}$ satisfy $\sum_{1 \leq j \leq N} \partial_{j} \psi(x) v_{j}=0$, then $\left.\sum_{1 \leq j, k \leq N} \partial_{j k}^{2} \psi(x) v_{j} v_{k}>0\right)$. It is well known that $\bar{\Omega}_{\lambda}$ is then convex in the usual sense (see e.g. [14]). Put

$$
\begin{gathered}
h(t, m)=\left(t, m+\left(t-t_{0}\right)\left|\psi^{\prime}(m)\right|^{-1} \psi^{\prime}(m)\right), \\
\Sigma_{\lambda}=\left\{h(t, m), t>t_{0}, \psi(m)=\lambda\right\},
\end{gathered}
$$

$V=\bigcup_{a<\lambda<b} \Sigma_{\lambda}$. It is well known (cf. e.g. [3]) that, because of our convexity assumptions, the equality $\varphi(h(t, m))=\psi(m)$ defines a $C^{\infty}(\bar{V})$ function $\varphi(t, x)$ which satisfies $\partial_{0} \varphi+\left|\partial_{x} \varphi\right|=0$ if $(t, x) \in V, \varphi\left(t_{0}, x\right)=\psi(x)$ if $\left(t_{0}, x\right) \in \bar{V}$. (Throughout all this paper we denote the closure of a set $\mathscr{E}$ by $\overline{\mathscr{E}}$ ).

Let $K_{1}, K_{2}$ be two compact subsets of $\mathbb{R}^{N}$ such that $a<\psi<b$ on $K_{1}$. The support of the "oscillatory part" (resp. "nonoscillatory part") of the initial data to be considered will be contained in $K_{1}$ (resp. $K_{2}$ ). Put $W=\{h(t, m), t>$ $\left.t_{0}, m \in K_{1}\right\}$. 
The first result deals with global existence of oscillatory approximate solutions of (2.1) for suitable initial data (see [5] for the construction of local oscillatory approximate solutions for first order quasilinear hyperbolic systems). We fix $\mu \in \mathbb{N}$ with $\mu \geq 2$.

Theorem 1. Assume that $N \geq 3$ is odd and that the null condition is satisfied if $N=3$. Also assume that $\zeta_{j}(x, \theta), 2 \leq j \leq \mu$, belong to $C^{\infty}\left(\mathbb{R}^{N} \times \mathbb{R}\right)$, vanish when $x \notin K_{1}$, are periodic of period $2 \pi$ with respect to $\theta$, and satisfy $\int_{0}^{2 \pi} \zeta_{j}(x, \theta) d \theta=0$ for all $x \in \mathbb{R}^{N}$. If $N \geq 5$, suppose that $\sup \left|\partial \zeta_{2} / \partial \theta\right| \leq$ $\delta$, where $\delta$ is a certain constant which is imposed by $\psi$ and the coefficients $f^{i j}$. Let $\omega_{j}^{k}, \quad 1 \leq j \leq \mu, k=0,1$, belong to $C_{0}^{\infty}\left(K_{2}\right)$. Then one can find $z_{j}(t, x, \theta) \in C^{\infty}\left(\left[t_{0},+\infty\left[\times \mathbb{R}^{N} \times \mathbb{R}\right), 1 \leq j \leq \mu\right.\right.$, and $\varepsilon_{0}>0$, with the following properties:

(i) $z_{1}$ is independent of $\theta$ for all $(t, x)$, and each $z_{j}, j \geq 2$, is independent of $\theta$ is $(t, x) \notin W$; so if $(t, x) \notin W$, we have $z_{j}(t, x, \theta) \equiv \hat{z}_{j}(t, x)$ with $\hat{z}_{j} \in C^{\infty}$;

(ii) $\partial_{\theta} z_{j}\left(t_{0}, x, \theta\right)=\zeta_{j}(x, \theta)$ if $2 \leq j \leq \mu$ and $(x, \theta) \in \mathbb{R}^{N} \times \mathbb{R}$, $\left.\partial_{0}^{k}\left(\int_{0}^{2 \pi} z_{j}(t, x, \theta) d \theta\right)\right|_{t=t_{0}}=\omega_{j}^{k}(x)$ if $1 \leq j \leq \mu, k=0,1$, and $x \in \mathbb{R}^{N}$;

(iii) if $\left.\varepsilon \in] 0, \varepsilon_{0}\right]$, if $z_{j}^{*}(t, x, \varepsilon)$ is equal to $z_{j}(t, x, \varphi(t, x) / \varepsilon)$ when $(t, x) \in$ $W$ and to $\hat{z}_{j}(t, x)$ when $(t, x) \notin W$, if $z^{\varepsilon}(t, x)=\sum_{1 \leq j \leq \mu} \varepsilon^{j} z_{j}^{*}(t, x, \varepsilon)$, and if $R^{\varepsilon}=\varepsilon^{-\mu}\left(\square z^{\varepsilon}-\sum_{0 \leq i, j \leq N} f^{i j}\left(z^{\varepsilon},\left(z^{\varepsilon}\right)^{\prime}\right) \partial_{i j}^{2} z^{\varepsilon}-f\left(z^{\varepsilon},\left(z^{\varepsilon}\right)^{\prime}\right)\right)$, then the following estimates hold with constants $C_{\alpha}$ independent of $\left.\left.\varepsilon \in\right] 0, \varepsilon_{0}\right]$ and of $(t, x) \in\left[t_{0},+\infty\left[\times \mathbb{R}^{N}:\right.\right.$

$$
\begin{gathered}
\left|\partial^{\alpha+\nu} z^{\varepsilon}(t, x)\right| \leq C_{\alpha} \varepsilon^{1-|\alpha|}(1+|t|+|x|)^{-(N-1) / 2}(1+|t-| x||)^{-(N-1) / 2} \text { if }|\nu| \leq 1 ; \\
\left|\partial^{\alpha} R^{\varepsilon}(t, x)\right| \leq C_{\alpha} \varepsilon^{-|\alpha|}(1+|t|+|x|)^{-(N+3) / 2}(1+|t-| x||)^{-(N+3) / 2} .
\end{gathered}
$$

Remark 2.1. The construction of $z^{\varepsilon}$ in the proof of Theorem 1 will determine $z_{j}, 1 \leq j \leq \mu-1$, and $\partial_{\theta} z_{\mu}$, but, as this proof will show, $\int_{0}^{2 \pi} z_{\mu}(t, x, \theta) d \theta$ will be very arbitrary.

Remark 2.2. When $N=3$, it is not necessary to impose a smallness condition on $\left|\partial \zeta_{2} / \partial \theta\right|$ because the null condition is assumed to hold; the same is true when $N>3$, if we assume that the nonlinear second order terms in (2.1) satisfy a condition analogous to the null condition. This will follow from the proof of Theorem 1.

Remark 2.3. The strong convexity assumptions and, if $N=3$, the null condition are important in Theorem 1; see Remark 4.3 below.

Starting from the global approximate solution $z^{\varepsilon}$ which will be constructed in Theorem 1, one can obtain a global exact solution of (2.1) with the same initial conditions and the same asymptotic behaviour as $z^{\varepsilon}$, as the next theorem shows.

Theorem 2. Let us keep the assumptions of Theorem 1, and assume that $\mu>$ $(N+3) / 2$. Then, if $\varepsilon_{1}$ is small enough and $\left.\left.\varepsilon \in\right] 0, \varepsilon_{1}\right],(2.1)$ has a global solution $\underline{z}^{\varepsilon}$ such that $\partial_{0}^{k} \underline{z}^{\varepsilon}=\partial_{0}^{k} z^{\varepsilon}$ if $k=0,1$ and $t=t_{0}$, and the following estimates hold:

$\left|\partial^{\alpha+\nu}\left(\underline{z}^{\varepsilon}-z^{\varepsilon}\right)(t, x)\right| \leq C_{\alpha} \varepsilon^{\mu-(N+1) / 2-|\alpha|}(1+|t|+|x|)^{-(N-1) / 2}(1+|t-| x||)^{-(N-1) / 2}$ 
if $|\alpha| \leq \mu-(N+1) / 2$ and $|\nu| \leq 1$, where the constant $C_{\alpha}$ is independent of $\left.\varepsilon \in] 0, \varepsilon_{1}\right]$ and of $(t, x) \in\left[t_{0},+\infty\left[\times \mathbb{R}^{N}\right.\right.$;

(ii)

$$
\iint_{t>t_{0}} \frac{\left|\partial^{\alpha+\nu}\left(\underline{z}^{\varepsilon}-z^{\varepsilon}\right)(t, x)\right|^{2}}{(1+|t|+|x|)^{2}(1+|t-| x||)^{2}} d t d x \leq C_{\alpha} \varepsilon^{2(\mu-|\alpha|)}
$$

if $|\alpha| \leq \mu$ and $|\nu| \leq 1$, where the constant $C_{\alpha}$ is independent of $\left.\left.\varepsilon \in\right] 0, \varepsilon_{1}\right]$.

Remark 2.4. In [5], Guès proved, for bounded time, the existence of solutions of Cauchy problems (for quasilinear first order symmetric hyperbolic systems) close to suitable approximate solutions. We shall make use of this result in the proof of Theorem 2.

Remark 2.5. The functions $z^{\varepsilon}, \underline{z}^{\varepsilon}$ to be obtained in the present paper and their gradients will be bounded by $C \varepsilon$, where $C$ is a constant. Cutting $f^{i j}$, $f$ off outside a neighbourhood of $(0,0)$ and taking $\varepsilon$ small, we see that it is enough to prove Theorems 1 and 2 when $f^{i j}, f \in C^{\infty}\left(\mathbb{R} \times \mathbb{R}^{N+1}\right)$ and so we shall henceforth assume that $f^{i j}, f \in C^{\infty}\left(\mathbb{R} \times \mathbb{R}^{N+1}\right)$.

Remark 2.6. Theorems 1 and 2 are time translation invariant, so that it is clearly enough to prove them for one value of $t_{0}$. However to economize notations, it will be convenient in subsequent constructions to assume from the beginning that $t_{0}$ is large enough; this is the reason why we have introduced $t_{0}$ in the formulation of $(2.1),(2.2)$.

\section{The KelVin tRANSFORM}

We are now going to transform (2.1), (2.2) into a finite time problem without introducing singularities in the partial differential equation. (Such a method was used in [1] and [4].) To achieve this we shall use the so-called Kelvin transform which was introduced in [13] for the study of decay properties of solutions of $\square z=0$ outside a star-shaped obstacle. Relevant properties for the study of (2.1) were described in [4]. Let us recall them quickly.

Write $C_{ \pm}=\left\{(t, x) \in \mathbb{R} \times \mathbb{R}^{N}, t \gtrless|x|\right\}$, and define the conformal inversion $J: C_{ \pm} \rightarrow C_{\mp}$ by $J(t, x)=(s, y)$, where $s=\left(|x|^{2}-t^{2}\right)^{-1} t, y=\left(|x|^{2}-t^{2}\right)^{-1} x$. Denote by $\eta$ the Minkowski metric; we have $\eta(v, w)=v_{0} w_{0}-\sum_{1 \leq j \leq N} v_{j} w_{j}$ if $v=\left(v_{0}, \ldots, v_{N}\right) \in \mathbb{R}^{N+1}$ and $w=\left(w_{0}, \ldots, w_{N}\right) \in \mathbb{R}^{N+1}$. Put $D_{0}=\partial / \partial s$, $D_{k}=\partial / \partial y_{k}$ if $1 \leq k \leq N, \sigma(s, y)=\left(s^{2}-|y|^{2}\right)^{1 / 2}, \square^{\prime}=D_{0}^{2}-\sum_{1 \leq j \leq N} D_{j}^{2}$, $D \Psi=\left(D_{0} \Psi, D_{1} \Psi, \ldots, D_{N} \Psi\right), D_{i j}^{2}=D_{i} D_{j}$. Sometimes we shall also write $D_{y} \Psi$ instead of $\left(D_{1} \Psi, \ldots, D_{N} \Psi\right)$, and $y_{0}$ for $s$. The following lemma holds.

Lemma 3.1 (see [4]). (1) $J=J^{-1}$ and $J$ is a conformal diffeomorphism for the Minkowski metric.

(2) If $p=(t, x)$ and $J(p)=(s, y)$, then $d J_{p}\left(\partial_{k}(p)\right)=K_{k}(J(p))$, where $K_{k}=\left(|y|^{2}-s^{2}\right) D_{k}+2 \beta_{k} y_{k}\left(s D_{0}+\sum_{1 \leq j \leq N} y_{j} D_{j}\right)$; here $\beta_{0}=1$ and $\beta_{k}=-1$ for $k>0$.

(3) If $z(t, x)$ is a smooth function, then

$$
\square z(J(s, y))=\sigma^{N+3}(s, y) \square^{\prime}\left(\sigma^{-(N-1)}(s, y) z(J(s, y))\right) .
$$

(4) If $Z(s, y)$ is a smooth function, and if $K_{k}, \beta_{k}$ are as in (2), then $K_{k}\left(\sigma^{N-1} Z\right)=\sigma^{N-1}\left(K_{k}+(N-1) \beta_{k} y_{k}\right) Z$. 
Put $Z(s, y)=\sigma^{-(N-1)}(s, y) z(t, x)$, where $(s, y)=J(t, x)$. The mapping $(t, x, z) \mapsto(s, y, Z)$ is called the Kelvin transform in [13]. If $N$ is odd and $\geq 3$, and if the null condition is satisfied when $N=3$, it is shown in [4] that the equation (which occurs in (2.1)) $\square z=\sum_{0 \leq i, j \leq N} f^{i j}\left(z, z^{\prime}\right) \partial_{i j}^{2} z+f\left(z, z^{\prime}\right)$, for $z(t, x),(t, x) \in U \subset C_{+}$, goes over to

$$
\square^{\prime} Z=\sum_{0 \leq i, j \leq N} g^{i j}(s, y, Z, D Z) D_{i j}^{2} Z+g(s, y, Z, D Z),
$$

where $(s, y) \in J(U) \subset C_{-}$. Here $g^{i j}$ and $g$ belong to $C^{\infty}\left(\mathbb{R} \times \mathbb{R}^{N} \times \mathbb{R} \times \mathbb{R}^{N+1}\right)$ (because we now assume that $f^{i j}, f \in C^{\infty}\left(\mathbb{R} \times \mathbb{R}^{N+1}\right)$, see Remark 2.4), $g^{i j}=$ $g^{j i}$ and $\left.g^{i j}(s, y, 0,0) \equiv \partial_{Z, P}^{\alpha} g(s, y, Z, P)\right|_{Z=P=0} \equiv 0$ if $|\alpha| \leq 1$. In fact the right-hand side of $(3.1)$ at point $(s, y)$ is equal to

$$
\sigma^{-(N+3)}(s, y)\left(\sum_{0 \leq i, j \leq N} f^{i j}\left(z, z^{\prime}\right) \partial_{i j}^{2} z+f\left(z, z^{\prime}\right)\right)(J(s, y)) .
$$

\section{Proof of Theorem 1}

First we make a time translation to prepare for the use of the Kelvin transform. Let $M>0$ be such that $|x| \leq M$ in $\Omega_{b} \cup K_{2}$, and let $t_{0}>M$ be chosen later. For proving Theorem 1 by means of the Kelvin transform, we shall find a suitable approximate oscillatory solution $Z^{\varepsilon}$ of (3.1) in $J\left(\left\{(t, x) \in C_{+}, t>\right.\right.$ $\left.\left.t_{0}\right\}\right)=\left\{(s, y) \in C_{-},|y|^{2}-s^{2}-t_{0}^{-1} s>0\right\}$, and pull it back to $C_{+}$by means of $J^{-1}=J$. Actually we shall construct $Z^{\varepsilon}$ in $\mathscr{D}=\left\{(s, y) \in \mathbb{R}^{N+1}, s<0\right.$, $\left.|y|^{2}-s^{2}-t_{0}^{-1} s>0\right\}$. This will immediately give the global estimates announced in Theorem 1; moreover, constructing approximate solutions defined beyond $\mathscr{D} \cap C_{-}$will also turn out to be useful for the proof of Theorem 2. The first step is to obtain the phase $\Phi$ of the oscillations of $Z^{\varepsilon}$. Define $H_{0}=\left\{(t, x) \in C_{+}, t^{2}-|x|^{2}-t_{0} t=0\right\}$, and for $m \in \Omega_{b} \backslash \bar{\Omega}_{a}$, put $h(t, m)=\left(t, m+\left(t-t_{0}\right)\left|\psi^{\prime}(m)\right|^{-1} \psi^{\prime}(m)\right)$ as before, $\gamma_{m}=\{h(t, m), t \in \mathbb{R}\}$. It follows from the results of $\S 4$ of [4] that the following holds when $\lambda \in] a, b[$ and $\psi(m)=\lambda:$ if $t_{0}>2 M$, then $\gamma_{m} \cap H_{0}$ is a single point; and if $t_{0}$ is large enough and $\widehat{\Omega}_{\lambda}$ is the bounded connected component (in the plane $s=-1 / t_{0}$ ) of the complement of $\bigcup_{m \in \partial \Omega_{\lambda}} J\left(\gamma_{m} \cap H_{0}\right)$, then $\widehat{\Omega}_{\lambda}$ is a bounded strongly convex open subset of the plane $s=-1 / t_{0}$, with $C^{\infty}$ boundary. Now put $\hat{\psi}(y)=\varphi(t, x)$ if $J(t, x)=\left(-1 / t_{0}, y\right), D \hat{\psi}(y)=\left(D_{1} \hat{\psi}(y), \ldots, D_{N} \hat{\psi}(y)\right)$, and define $\hat{h}(s, \hat{m})=$ $\left(s, \hat{m}+\left(s+1 / t_{0}\right)|D \hat{\psi}(\hat{m})|^{-1} D \hat{\psi}(\hat{m})\right), \widehat{V}=\left\{\hat{h}(s, \hat{m}), s>-1 / t_{0}, \hat{m} \in \widehat{\Omega}_{b} \backslash \widehat{\widehat{\Omega}}_{a}\right\}$. From standard properties of characteristic surfaces of the d'Alembertian (cf. e.g. [3]), it follows that the equation $\widehat{\Phi}(s, \hat{h}(s, \hat{m}))=\hat{\psi}(\hat{m})$ defines a function $\widehat{\Phi} \in C^{\infty}(\overline{\widehat{V}})$ with

$$
D_{0} \hat{\boldsymbol{\Phi}}+\left|D_{y} \hat{\boldsymbol{\Phi}}\right|=0 \text { in } \widehat{V} \quad \text { and } \quad \hat{\Phi}\left(-1 / t_{0}, y\right)=\hat{\psi}(y) \text { if } y \in \widehat{\Omega}_{b} \backslash \overline{\widehat{\Omega}}_{a} .
$$

Define $\widetilde{\Phi}: J(V) \rightarrow \mathbb{R}$ by $\widetilde{\Phi}=\varphi \circ J^{-1}$. A simple computation gives that $\left(D_{0} \widetilde{\Phi}\right)^{2}-\left|D_{y} \widetilde{\Phi}\right|^{2}=0$ and $D_{0} \widetilde{\Phi}<0$ in $J(V)$, so that $D_{0} \widetilde{\Phi}+\left|D_{y} \widetilde{\Phi}\right|=0$ there. 
Furthermore since

$$
d J\left(\partial_{0} \varphi \cdot \partial_{0}-\sum_{1 \leq i \leq N} \partial_{i} \varphi \cdot \partial_{i}\right)=\left(s^{2}-|y|^{2}\right)^{2}\left(D_{0} \widetilde{\Phi} \cdot D_{0}-\sum_{1 \leq i \leq N} D_{i} \widetilde{\Phi} \cdot D_{i}\right),
$$

$J(V)$ is a union of open intervals of integral curves of $D_{0} \widetilde{\Phi} \cdot D_{0}-\sum_{1 \leq i \leq N} D_{i} \widetilde{\Phi}$. $D_{i}$, having one endpoint in $J\left(\left\{t_{0}\right\} \times\left(\Omega_{b} \backslash \bar{\Omega}_{a}\right)\right)$. From standard facts of the Hamilton-Jacobi method for solving (4.1) (see e.g. [3]), it easily follows that $\widehat{V} \cap C_{-}=\left\{(s, y) \in J(V), s>-1 / t_{0}\right\}$, and that $\widehat{\Phi}=\widetilde{\Phi}$ in $\widehat{V} \cap C_{-}$. Put $V^{\prime}=\widehat{V} \cup J(V)$. If we now define $\Phi$ to be equal to $\hat{\Phi}$ in $\widehat{V}$ and to $\widetilde{\Phi}$ in $\left\{(s, y) \in J(V), s \leq-1 / t_{0}\right\}$, we have thus proved the following lemma.

Lemma 4.1. There exists $\Phi \in C^{\infty}\left(\overline{V^{\prime}}\right)$ such that $D_{0} \Phi+\left|D_{y} \Phi\right|=0$ in $V^{\prime}$ and $\Phi=\varphi \circ J^{-1}$ in $J(V)$.

We are now ready to start the construction of the announced oscillatory approximate solution $Z^{\varepsilon}$ of $(3.1)$ such that $\left(\sigma^{N-1} Z^{\varepsilon}\right) \circ J$ will be the sought approximate solution $z^{\varepsilon}$ of Theorem 1 . WKB local approximate solutions for first order quasilinear hyperbolic systems have been constructed in [5], but treating the second order scalar case directly without reducing to a first order system gives a somewhat simpler and more explicit construction. Put $V_{0}^{\prime}=\left\{(s, y) \in V^{\prime}\right.$, $s<0\}$. We shall construct an approximate solution of (3.1) in $\mathscr{D}$ with oscillations (of phase $\Phi$ ) contained in $V_{0}^{\prime}$. To simplify the writing, it is convenient to extend the function $\Phi$ given by Lemma 4.1 to $\mathscr{D}$ as a $C^{\infty}(\overline{\mathscr{D}})$ function, which we also denote by $\Phi$. With this notation, we shall find a $C^{\infty}$ approximate solution $Z^{\varepsilon}(s, y), \varepsilon>0$, of the following form:

$$
Z^{\varepsilon}(s, y)=\varepsilon Z_{1}(s, y)+\sum_{2 \leq j \leq \mu} \varepsilon^{j} Z_{j}\left(s, y, \frac{\Phi(s, y)}{\varepsilon}\right)
$$

if $(s, y) \in \overline{\mathscr{D}}$, where $Z_{1} \in C^{\infty}(\overline{\mathscr{D}}) ; Z_{j}(s, y, \theta), 2 \leq j \leq \mu$, are $C^{\infty}$ functions of $(s, y) \in \bar{D}$ and $\theta \in \mathbb{R}$, periodic of period $2 \pi$ with respect to $\theta$, and independent of $\theta$ if $(s, y) \notin V_{0}^{\prime}$. Of course (4.2) does not depend on the value of $\Phi$ in $\mathscr{D} \backslash V_{0}^{\prime}$ but the extension of $\Phi$ makes the righthand side of (4.2) well defined for all $(s, y) \in \overline{\mathscr{D}}$. To simplify the notations in (4.2), we shall often write $Z_{1}(s, y, \theta)$ instead of $Z_{1}(s, y)$ and so $Z^{\varepsilon}(s, y)=\sum_{1 \leq j \leq \mu} \varepsilon^{j} Z_{j}(s, y, \Phi(s, y) / \varepsilon)$. Put

$$
\begin{gathered}
M_{j}(s, y, \theta)=\left(Z_{j}(s, y, \theta),\left(D_{k} Z_{j}(s, y, \theta)+D_{k} \Phi(s, y) \cdot \partial_{\theta} Z_{j+1}(s, y, \theta)\right)_{0 \leq k \leq N}\right), \\
1 \leq j \leq \mu,
\end{gathered}
$$

with $Z_{\mu+1} \equiv 0$. If $p \in \mathbb{R}^{N+2}$, if $h(s, y, p)$ is a $C^{\infty}$ function such that $h(s, y, 0) \equiv 0$, and if $\nu \in \mathbb{N} \backslash\{0\}$, a Taylor expansion in powers of $\varepsilon$ gives

$$
\begin{aligned}
& h\left(s, y, \sum_{1 \leq j \leq \mu} \varepsilon^{j} M_{j}(s, y, \theta)\right) \\
& =\sum_{1 \leq k \leq \nu} \varepsilon^{k} G_{k}(h ; s, y, \theta)+\varepsilon^{\nu+1} \mathscr{G}_{\nu+1}(h ; s, y, \theta, \varepsilon),
\end{aligned}
$$


where $(s, y, \theta) \mapsto G_{k}(h ; s, y, \theta)$ and $(s, y, \theta, \varepsilon) \mapsto \mathscr{G}_{\nu+1}(h ; s, y, \theta, \varepsilon)$ are $C^{\infty}$ functions (depending on $Z_{j}, D_{k} Z_{j}, \partial_{\theta} Z_{j}, D_{k} \Phi, 1 \leq j \leq \mu, 0 \leq k \leq$ $N)$, periodic of period $2 \pi$ with respect to $\theta$. Henceforth, $g^{i j}(s, y, Z, P)$ and $g(s, y, Z, P)$ will be the functions appearing in (3.1). It is convenient to introduce the following notations, which will be used repeatedly in this section:

$$
\begin{gathered}
X=D_{0} \Phi \cdot D_{0}-\sum_{1 \leq j \leq N} D_{j} \Phi \cdot D_{j}, \quad c^{i j}(s, y)=\frac{\partial g^{i j}}{\partial Z}(s, y, 0,0), \\
c^{i j k}(s, y)=\frac{\partial g^{i j}}{\partial P_{k}}(s, y, 0,0), \\
Q=\sum_{0 \leq i, j \leq N} D_{i} \Phi \cdot D_{j} \Phi \cdot\left(c^{i j}+\sum_{0 \leq k \leq N} c^{i j k} D_{k}\right), \\
A=\sum_{0 \leq i, j, k \leq N} c^{i j k} D_{i} \Phi \cdot D_{j} \Phi \cdot D_{k} \Phi .
\end{gathered}
$$

Now we may write, since $Z_{1}$ is independent of $\theta$ :

$$
D_{i j}^{2} Z^{\varepsilon}(s, y)=\sum_{0 \leq k \leq \mu} \varepsilon^{k} S_{i j k}\left(s, y, \frac{\Phi(s, y)}{\varepsilon}\right),
$$

with

$$
\begin{aligned}
S_{i j k}(s, y, \theta)=\left(D_{i} \Phi\right. & \cdot D_{j} \Phi \cdot \partial_{\theta}^{2} Z_{k+2} \\
& \left.+\left(D_{j} \Phi \cdot D_{i}+D_{i} \Phi \cdot D_{j}+D_{i j}^{2} \Phi\right) \partial_{\theta} Z_{k+1}+D_{i j}^{2} Z_{k}\right)(s, y, \theta)
\end{aligned}
$$

(where $Z_{n}$ is to be replaced by 0 if $n>\mu$ or if $n=0$ ). On the other hand we have

$$
\begin{gathered}
\sum_{\substack{0 \leq i, j \leq N \\
0 \leq k \leq \mu}} \eta^{i j} \varepsilon^{k} S_{i j k}-\sum_{0 \leq i, j \leq N} g^{i j}\left(s, y, \sum_{1 \leq l \leq \mu} \varepsilon^{l} M_{l}\right) \sum_{0 \leq k \leq \mu} \varepsilon^{k} S_{i j k} \\
\quad-g\left(s, y, \sum_{1 \leq l \leq \mu} \varepsilon^{l} M_{l}\right) \\
=\sum_{1 \leq k \leq \mu-1} \varepsilon^{k} F_{k}+\varepsilon^{\mu} R_{\mu} \quad \text { if }(s, y) \in \mathscr{D}, \theta \in \mathbb{R}, \varepsilon>0
\end{gathered}
$$

where $F_{k}, 1 \leq k \leq \mu-1$, is the coefficient of $\varepsilon^{k}$ in the Taylor expansion (in powers of $\varepsilon$ ) of the left-hand side of (4.5). Making use of an expansion of type (4.3) for $g^{i j}$ and $g$ (with $\nu=\mu-1$ ) and grouping the coefficients of the same powers of $\varepsilon$, we find that (with $X, Q, A$ as defined just before (4.4)):

$$
\begin{aligned}
F_{1}(s, y, \theta)= & \left(2 X+\square^{\prime} \Phi-\left(Q Z_{1}+A \partial_{\theta} Z_{2}\right) \partial_{\theta}\right) \partial_{\theta} Z_{2}(s, y, \theta) \\
& +\square^{\prime} Z_{1}(s, y), \\
F_{k}(s, y, \theta)= & \left(2 X+\square^{\prime} \Phi-\left(Q Z_{1}+A \partial_{\theta} Z_{2}\right) \partial_{\theta}-A \partial_{\theta}^{2} Z_{2}\right) \partial_{\theta} Z_{k+1}(s, y, \theta) \\
& +\left(\square^{\prime} Z_{k}-Q Z_{k} \cdot \partial_{\theta}^{2} Z_{2}\right)(s, y, \theta) \\
+ & \mathscr{F}_{k}\left(s, y,\left(\partial_{\theta}^{l} D^{\alpha} Z_{j}(s, y, \theta)\right)_{l+|\alpha| \leq 2,1 \leq j \leq k(l, \alpha)}\right)
\end{aligned}
$$


if $2 \leq k \leq \mu-1$, where $\mathscr{F}_{k}$ is a $C^{\infty}$ function (depending on the derivatives of order 1 and 2 of $\Phi)$ such that $v \mapsto \mathscr{F}_{k}(s, y, v)$ vanishes of order 2 when $v=0 ; \alpha=\left(\alpha_{0}, \alpha_{1}, \ldots, \alpha_{N}\right)$ and $D^{\alpha}=D_{0}^{\alpha_{0}} D_{1}^{\alpha_{1}} \ldots D_{N}^{\alpha_{N}} ; k(l, \alpha)=k-|\alpha|$ if $l+|\alpha|>0, k(0,0)=k-1$;

$$
\begin{aligned}
R_{\mu}(s, y, \theta, \varepsilon) & =\sum_{\substack{0 \leq i, j \leq N \\
k \geq \mu}} \eta^{i j} \varepsilon^{k-\mu} S_{i j k}(s, y, \theta) \\
& -\sum_{\substack{0 \leq i, j \leq N \\
k+l \geq \mu}} \varepsilon^{k+l-\mu} G_{k}\left(g^{i j} ; s, y, \theta\right) S_{i j l}(s, y, \theta) \\
& -\sum_{\substack{0 \leq i, j \leq N \\
l \geq 0}} \varepsilon^{l} \mathscr{G}_{\mu}\left(g^{i j} ; s, y, \theta, \varepsilon\right) S_{i j l}(s, y, \theta)-\mathscr{G}_{\mu}(g ; s, y, \theta, \varepsilon) .
\end{aligned}
$$

From (4.5) it follows that

$$
\begin{aligned}
\square^{\prime} Z^{\varepsilon} & -\sum_{0 \leq i, j \leq N} g^{i j}\left(s, y, Z^{\varepsilon}, D Z^{\varepsilon}\right) D_{i j}^{2} Z^{\varepsilon}-g\left(s, y, Z^{\varepsilon}, D Z^{\varepsilon}\right) \\
& =\sum_{1 \leq k \leq \mu-1} \varepsilon^{k} F_{k}\left(s, y, \frac{\Phi(s, y)}{\varepsilon}\right)+\varepsilon^{\mu} R_{\mu}\left(s, y, \frac{\Phi(s, y)}{\varepsilon}, \varepsilon\right)
\end{aligned}
$$

if $(s, y) \in \mathscr{D}$. Assume that there exists a bounded subset $\mathscr{K}$ of $\mathscr{D}$ such that $Z_{j}(s, y, \theta)=0$ if $1 \leq j \leq \mu,(s, y) \notin \mathscr{K}, \theta \in \mathbb{R}$. Then, using (4.8), one can easily check that for each $\varepsilon_{0}>0$, one can find $C_{\alpha}>0$ such that

$$
\left|D_{s y}^{\alpha}\left(R_{\mu}\left(s, y, \frac{\Phi(s, y)}{\varepsilon}, \varepsilon\right)\right)\right| \leq C_{\alpha} \varepsilon^{-|\alpha|}
$$

$$
\text { if } \alpha \in \mathbb{N}^{N+1},(s, y) \in \mathscr{D} \text {, and } 0<\varepsilon \leq \varepsilon_{0} .
$$

We shall now choose $Z_{1}, \ldots, Z_{\mu-1}, \partial_{\theta} Z_{\mu}$ in such a way that $F_{k}(s, y, \theta) \equiv 0$ for $k=1, \ldots, \mu-1$. (Equations corresponding to $F_{k}(s, y, \theta) \equiv 0,1 \leq k \leq$ $\mu-1$, have been obtained by Guès in [5] for first order hyperbolic systems, but working directly with the second order equation without reducing to a system gives at once conditions which are simpler to study.)

Solving $F_{1}(s, y, \theta)=0$. Since we want $Z_{2}(s, y, \theta)$ to be periodic of period $2 \pi$ with respect to $\theta,(4.6)$ shows that we must have $\int_{0}^{2 \pi} F_{1}(s, y, \theta) d \theta=$ $2 \pi \square^{\prime} Z_{1}(s, y)$. Hence to satisfy the equation $F_{1}(s, y, \theta)=0$ with a suitable $Z_{2}$, we must impose the condition $\square^{\prime} Z_{1}(s, y)=0$. We first examine the way we shall impose this condition. Put $H=\left\{(s, y) \in C_{-},|y|^{2}-s^{2}-t_{0}^{-1} s=0\right\}$. To simplify notations in the sequel, it is convenient to introduce the functions $\tilde{\omega}_{j}^{i}(t, x), \tilde{\zeta}_{k}(t, x, \theta), i=0,1,1 \leq j \leq \mu, 2 \leq k \leq \mu$, defined by $\widetilde{\omega}_{j}^{i}(t, x) \equiv$ $\omega_{j}^{i}(x), \tilde{\zeta}_{k}(t, x, \theta) \equiv \zeta_{k}(x, \theta)$, with $\omega_{j}^{i}, \zeta_{k}$ as in the statement of Theorem 1. Let $\tilde{Z}_{1}(s, y)$ be the solution of the Cauchy problem

$$
\begin{gathered}
\square^{\prime} \tilde{Z}_{1}(s, y)=0 \quad \text { if }(s, y) \in \mathscr{D} \cap C_{-}, \\
\left(K_{0}+(N-1) s\right)^{j} \tilde{Z}_{1}(s, y)=\left(s^{2}-|y|^{2}\right)^{-(N-1) / 2}\left(\tilde{\omega}_{1}^{j} \circ J\right)(s, y) \\
\text { if } j=0,1 \text { and }(s, y) \in H .
\end{gathered}
$$


(4.11), (4.12) determine $\widetilde{Z}_{1}$ in $C_{-}$; however as already pointed out before, we want to construct an approximate solution of (3.1) in $\mathscr{D}$. So let us describe now an extension procedure which will be useful. Until the end of this paper, we shall put $r=\left(t_{0}-M\right)^{-1}$, where $M$ and $t_{0}>M$ are as in the beginning of this section. A simple computation shows that $\tilde{\omega}_{j}^{i}(J(s, y)) \equiv 0 \equiv \tilde{\zeta}_{k}(J(s, y), \theta)$ if $0 \leq i \leq 1,1 \leq j \leq \mu, 2 \leq k \leq \mu,(s, y) \in H,|y| \geq s+r$, and $\theta \in \mathbb{R}$. Therefore $\widetilde{Z}_{1}(s, y)=0$ if $|y| \geq s+r$ and $(s, y) \in \mathscr{D} \cap C_{-}$. Now let $r_{1}>0$ be so large that $|y| \leq r_{1}$ if $(s, y) \in H$ and $|y| \leq s+r$. Put $r_{2}=\left(r_{1}^{2}+1 / 4 t_{0}^{2}\right)^{1 / 2}$ and choose $\Theta \in C^{\infty}([0,+\infty[,[0,1])$ such that $\Theta(\lambda)=1$ if $\lambda \leq 2 r_{2}$ and $\Theta(\lambda)=0$ if $\lambda \geq 3 r_{2}$. Define $\widetilde{\Theta}(\lambda)=\int_{0}^{\lambda} \Theta(\tilde{\lambda}) d \tilde{\lambda}$ if $\lambda \geq 0$, $L(y)=\widetilde{\boldsymbol{\Theta}}\left(\left(|y|^{2}+1 / 4 t_{0}^{2}\right)^{1 / 2}\right)+1 / 2 t_{0}, H^{*}=\left\{(s, y) \in \mathbb{R}^{N+1}, s=-L(y)\right\}$. Then it is clear that $H^{*} \cap E=H \cap E$ if $E$ is a suitable neighbourhood (in $\mathbb{R}^{N+1}$ ) of the cone $\left\{(s, y) \in \mathbb{R}^{N+1},|y| \leq s+r\right\}$. Notice that $H^{*}$ is spacelike for $\square^{\prime}$. If $v$ is a function on $H$ such that $v(s, y)=0$ when $|y| \geq s+r$ and $(s, y) \in H$, we shall define the function $v^{*}$ on $H^{*}$ in the following way: $v^{*}(s, y)=v(s, y)$ if $(s, y) \in H^{*}$ and $|y| \leq s+r, v^{*}(s, y)=0$ if $(s, y) \in H^{*}$ and $|y|>s+r$. Let $Z_{1}(s, y)$ be the solution of the Cauchy problem

$$
\begin{aligned}
\square^{\prime} Z_{1}(s, y) & =0 \quad \text { if }(s, y) \in \mathscr{D}, \\
\left(K_{0}+(N-1) s\right)^{j} Z_{1}(s, y) & =\left(s^{2}-|y|^{2}\right)^{-(N-1) / 2}\left(\tilde{\omega}_{1}^{j} \circ J\right)^{*}(s, y) \\
& \text { if } j=0,1 \text { and }(s, y) \in H^{*} .
\end{aligned}
$$

Of course $Z_{1}=\widetilde{Z}_{1}$ in $\mathscr{D} \cap C_{-}$and $Z_{1}(s, y)=0$ if $(s, y) \in \mathscr{D}$ and $|y| \geq s+r$. Consider then the Cauchy problem (where $X, Q, A$ are the same as in (4.6)):

$$
\begin{gathered}
\left(2 X+\square^{\prime} \Phi-\left(Q Z_{1}+A w_{2}\right) \partial_{\theta}\right) w_{2}(s, y, \theta)=0 \quad \text { if }(s, y) \in V_{0}^{\prime}, \theta \in \mathbb{R} ; \\
w_{2}(s, y, \theta)=\left(s^{2}-|y|^{2}\right)^{-(N-1) / 2} \tilde{\zeta}_{2}(J(s, y), \theta) \\
\quad \text { if }(s, y) \in J\left(\left\{t_{0}\right\} \times\left(\Omega_{b} \backslash \bar{\Omega}_{a}\right)\right), \quad \theta \in \mathbb{R} .
\end{gathered}
$$

In the study of (4.15), (4.16) we shall make use of the following lemma.

Lemma 4.2. Assume that in (2.1), we have $f^{i j}(z, p)=b \eta^{i j} z+\sum_{0 \leq k \leq N} b^{i j k} p_{k}+$ $\mathscr{O}\left(z^{2}+|p|^{2}\right)$ as $(z, p) \rightarrow(0,0)$ in $\mathbb{R} \times \mathbb{R}^{N}$, where $p=\left(p_{0}, p_{1}, \ldots, p_{N}\right)$, $\eta^{00}=1, \eta^{i i}=-1$ if $i>0, \eta^{i j}=0$ if $i \neq j, b \in \mathbb{R}, b^{i j k} \in \mathbb{R}$, and $\sum_{0 \leq i, j, k \leq N} b^{i j k} q_{i} q_{j} q_{k}=0$ for all $q=\left(q_{0}, q_{1}, \ldots, q_{N}\right) \in \mathbb{R}^{N+1}$ such that $q_{0}^{2}=$ $\sum_{1 \leq k \leq N} q_{k}^{2}$ (if $N=3$, this is just the null condition for second order terms). Then $A=0$.

Proof of Lemma 4.2. A straightforward computation shows that

$$
\begin{aligned}
\sum_{0 \leq i, j \leq N} & g^{i j}(s, y, Z, D Z) D_{i j}^{2} Z \\
= & \sigma^{N-1} b Z \cdot \square^{\prime} Z+\sigma^{N-5} \sum_{0 \leq i, j, k \leq N} b^{i j k}\left(K_{k}+(N-1) \beta_{k} y_{k}\right) Z \\
& \cdot\left(K_{i} K_{j} Z+P_{i j}(s, y, D) Z\right)+\sum_{|\alpha| \leq 2} R_{\alpha}(s, y, Z, D Z) D^{\alpha} Z,
\end{aligned}
$$


where $\sigma, K_{i}$ are the same as in $\S 3, P_{i j}$ are linear first order differential operators with $C^{\infty}$ coefficients, $D^{\alpha}=D_{0}^{\alpha_{0}} D_{1}^{\alpha_{1}} \cdots D_{N}^{\alpha_{N}}, R_{\alpha} \in C^{\infty}$, and

$$
\left.\partial_{Z P}^{\gamma} R_{\alpha}(s, y, Z, P)\right|_{Z=P=0}=0 \quad \text { if }|\gamma| \leq 1 \text {. }
$$

It follows that

$$
c^{m n l}=\sigma^{N-5} \sum_{0 \leq i, j, k \leq N} b^{i j k} A_{k l} A_{i m} A_{j n}, \quad 0 \leq m, n, l \leq N,
$$

if $A_{i j}=\left(|y|^{2}-s^{2}\right) \delta_{i j}+2 \beta_{i} y_{i} y_{j}$, where $\delta_{i j}=1$ if $i=j$ and $\delta_{i j}=0$ if $i \neq j$. Now, with $Y=\left(Y_{0}, Y_{1}, \ldots, Y_{N}\right) \in \mathbb{R}^{N+1}$, put $B=\sum_{0 \leq k \leq N} \beta_{k} y_{k} \partial / \partial Y_{k}$, $\kappa=\sum_{0 \leq k \leq N} y_{k} Y_{k}$. An explicit computation using (4.17) shows that

$$
\begin{aligned}
& \sum_{0 \leq m, n, l \leq N} c^{m n l} Y_{m} Y_{n} Y_{l} \\
& \quad=\sigma^{N-5}\left(-\sigma^{6}+2 \sigma^{4} \kappa B-2 \sigma^{2} \kappa^{2} B^{2}+\frac{4}{3} \kappa^{3} B^{3}\right)\left(\sum_{0 \leq i, j, k \leq N} b^{i j k} Y_{i} Y_{j} Y_{k}\right) .
\end{aligned}
$$

The assumption on $b^{i j k}$ implies that $\sum_{0 \leq i, j, k \leq N} b^{i j k} Y_{i} Y_{j} Y_{k}=\eta(Y, Y) \Psi(Y)$, where $\eta$ is the Minkowski metric and $\Psi$ is a linear form (this can be checked easily by induction on $N$ ). Introducing this in the right-hand side of (4.18), we obtain after some easy computations that the right-hand side of (4.18) vanishes if $\eta(Y, Y)=0$; hence so does the left-hand side of (4.18). Choosing $Y=$ $D \Phi(s, y)$, we therefore obtain that $\sum_{0 \leq m, n, l \leq N} c^{m n l} D_{m} \Phi \cdot D_{n} \Phi \cdot D_{l} \Phi=0$ since $\left(D_{0} \Phi\right)^{2}=\sum_{1 \leq j \leq N}\left(D_{j} \Phi\right)^{2}$. This proves Lemma 4.2.

To study (4.15), (4.16), it is convenient to introduce new coordinates which will straighten out the integral curves of $X$. Let $\gamma_{m}, \hat{h}(s, \hat{m})$ be as at the beginning of the present section, and put $\hat{\gamma}_{\hat{m}}=\{\hat{h}(s, \hat{m}), s \in \mathbb{R}\}$. From standard properties of solutions of Hamilton-Jacobi equations (see e.g. [3]) it follows that $D_{0} \Phi=-|D \hat{\psi}(\hat{m})|$ at each point of $\hat{\gamma}_{\hat{m}} \cap V^{\prime}$; hence $s \mapsto \hat{h}(s, \hat{m})$ (restricted to those values of $s$ for which $\left.\hat{h}(s, \hat{m}) \in V^{\prime}\right)$ is the integral curve of $\left(D_{0} \Phi\right)^{-1} X$ through $\left(-1 / t_{0}, \hat{m}\right)$. In $\overline{V^{\prime}}$ new coordinates $(s, \hat{m})$ can be defined by the relation $\hat{h}(s, \hat{m})=(s, y)$ : when $s \geq-1 / t_{0}$, this follows from the convexity properties of $\hat{\psi}$ (see e.g. [3] for consequences of such convexity properties); when $s \leq-1 / t_{0}$, this can be checked using the conformal inversion $J$ (which transforms $\partial_{0} \varphi \cdot \partial_{0}-\sum_{1 \leq j \leq N} \partial_{j} \varphi \cdot \partial_{j}$ into $\left(s^{2}-|y|^{2}\right)^{2}\left(D_{0} \Phi \cdot D_{0}-\sum_{1 \leq j \leq N} D_{j} \Phi \cdot D_{j}\right)$ and so maps sets $\left\{(t, x) \in \gamma_{n}, t>t_{0}\right\}, n \in \Omega_{b} \backslash \bar{\Omega}_{a}$, onto sets $\hat{\gamma}_{\hat{n}} \cap \mathscr{D} \cap C_{-}$, $\hat{n} \in \widehat{\Omega}_{b} \backslash \overline{\boldsymbol{\Omega}}_{a}$, as is easily verified). Now, if $\hat{m} \in \overline{\widehat{\Omega}}_{b} \backslash \widehat{\Omega}_{a}, \hat{\gamma}_{\hat{m}}$ intersects $H$ exactly once and transversally, so $H \cap \overline{V^{\prime}}$ is given by an equation $s=\mathscr{S}(\hat{m}), \mathscr{S} \in C^{\infty}$. If we put $\tilde{\chi}(s, \hat{m})=\left(|D \hat{\psi}(\hat{m})|^{-1}(s-\mathscr{S}(\hat{m})), \hat{m}\right), \chi=\tilde{\chi} \circ h^{-1}$, we can easily check that $(\tilde{s}, \tilde{y})=\chi(s, y)$ is a $C^{\infty}$ diffeomorphism defined in $\overline{V^{\prime}}$, such that $\left.\chi\left(V^{\prime}\right)=\right] 0,+\infty\left[\times\left(\widehat{\boldsymbol{\Omega}}_{b} \backslash \overline{\widehat{\Omega}}_{a}\right), \chi\left(\overline{V^{\prime}} \cap H\right)=\{0\} \times\left(\overline{\widehat{\Omega}}_{b} \backslash \widehat{\boldsymbol{\Omega}}_{a}\right)\right.$, and $d \chi(X)=-\partial / \partial \tilde{s}$ in $\chi\left(\overline{V^{\prime}}\right)$. Put $\tilde{w}_{2}(\tilde{s}, \tilde{y}, \theta)=w_{2}\left(\chi^{-1}(\tilde{s}, \tilde{y}), \theta\right), E_{1}(\tilde{s}, \tilde{y})=\frac{1}{2}\left(\square^{\prime} \Phi\right)\left(\chi^{-1}(\tilde{s}, \tilde{y})\right)$, $E_{2}(\tilde{s}, \tilde{y})=\frac{1}{2}\left(Q Z_{1}\right)\left(\chi^{-1}(\tilde{s}, \tilde{y})\right), \tilde{A}(\tilde{s}, \tilde{y})=\frac{1}{2} A\left(\chi^{-1}(\tilde{s}, \tilde{y})\right)$. Then (4.15) becomes

$$
\left(\partial_{\tilde{s}}-E_{1}+\left(E_{2}+\tilde{A} \tilde{w}_{2}\right) \partial_{\theta}\right) \tilde{w}_{2}(\tilde{s}, \tilde{y}, \theta)=0 \quad \text { if }(\tilde{s}, \tilde{y}) \in \chi\left(V_{0}^{\prime}\right), \theta \in \mathbb{R}
$$


and (4.16) gives

$$
\tilde{w}_{2}(0, \tilde{y}, \theta)=\tilde{\xi}_{2}(\tilde{y}, \theta) \quad \text { if } \tilde{y} \in \widehat{\Omega}_{b} \backslash \overline{\boldsymbol{\Omega}}_{a}, \theta \in \mathbb{R},
$$

where $\tilde{\xi}_{2}(\tilde{y}, \theta)=\left(s^{2}-|y|^{2}\right)^{-(N-1) / 2} \tilde{\zeta}_{2}(J(s, y), \theta)$ with $(s, y)=\chi^{-1}(0, \tilde{y})$, so that $\tilde{\xi}_{2}(\tilde{y}, \theta)=0$ for every $\theta$ if $\tilde{y}$ does not belong to a certain compact subset of $\widehat{\Omega}_{b} \backslash \widehat{\widehat{\Omega}}_{a}$. Let $E(s, \tilde{y}) \in C^{\infty}\left(\left[0,+\infty\left[\times\left(\widehat{\widehat{\Omega}}_{b} \backslash \widehat{\Omega}_{a}\right)\right)\right.\right.$ satisfy $\partial_{\tilde{s}} E=-E_{1}$ in $\left[0,+\infty\left[\times\left(\widehat{\widehat{\Omega}}_{b} \backslash \widehat{\Omega}_{a}\right)\right.\right.$ and put $w=e^{E} \tilde{w}_{2}, E_{3}=e^{-E} \tilde{A}$. Then (4.19) becomes

$$
\left(\partial_{\tilde{s}}+\left(E_{2}+E_{3} w\right) \partial_{\theta}\right) w(\tilde{s}, \tilde{y}, \theta)=0 \quad \text { if }(\tilde{s}, \tilde{y}) \in \chi\left(V_{0}^{\prime}\right), \theta \in \mathbb{R},
$$

while (4.20) gives

$$
w(0, \tilde{y}, \theta)=e^{E(0, \tilde{y})} \tilde{\xi}_{2}(\tilde{y}, \theta) \quad \text { if } \tilde{y} \in \widehat{\Omega}_{b} \backslash \overline{\widehat{\Omega}}_{a}, \theta \in \mathbb{R} .
$$

We may now discuss (4.15), (4.16) (or the equivalent equations (4.21), (4.22)). To solve (4.21), (4.22), we may use the classical method of characteristics (see e.g. [2]). Put

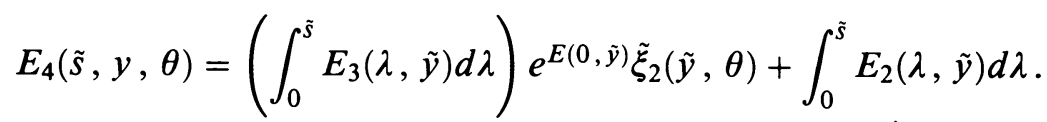

The characteristics (for equality (4.21)) are parametrized by $\Lambda_{\tilde{y}, \theta}: \tilde{s} \mapsto$ $\left(\tilde{s}, \tilde{y}, \theta+E_{4}(\tilde{s}, y, \theta)\right)$ and $w\left(\Lambda_{\tilde{y}, \theta}(\tilde{s})\right)$ does not depend on $\tilde{s}$ if $w$ is a solution of (4.21). Let $\tilde{s}_{1}$ be such that $\tilde{s} \leq \tilde{s}_{1}$ on $\chi\left(V_{0}^{\prime}\right)$. It is clear that there exists $\delta>0$ such that the following holds: if $\tilde{s} \in\left[0, \tilde{s}_{1}\right], \tilde{y} \in \widehat{\Omega}_{b} \backslash \widehat{\widehat{\Omega}}_{a}$ and $\left|\partial_{\theta} \tilde{\xi}_{2}\right| \leq \delta$, then $\partial_{\theta} E_{4} \geq-\frac{1}{2}$. In that case, the mapping $\Lambda:(\tilde{s}, \tilde{y}, \theta) \mapsto \Lambda_{\tilde{y}, \theta}(\tilde{s})$ can be inverted and therefore the equality

$$
w(\Lambda(\tilde{s}, \tilde{y}, \theta))=e^{E(0, \tilde{y})} \tilde{\xi}_{2}(\tilde{y}, \theta)
$$

defines the unique $C^{\infty}$ solution of (4.21), (4.22). It follows at once that (4.15), (4.16) has a unique $C^{\infty}$ solution if $\sup \left|\partial_{\theta} \zeta_{2}\right|$ is small enough. If $A=0$, then $E_{3}=0,(4.15)$ and (4.21) are linear, and $\partial_{\theta} E_{4}=0$; so if $A=0$ it follows that $(4.15),(4.16)$ has a unique $C^{\infty}$ solution whatever the size of sup $\left|\partial_{\theta} \zeta_{2}\right|$. Now because of the assumptions of the present paper, the condition $A=0$ is satisfied in case $N=3$; indeed we assume then that the null condition is fulfilled in (2.1) and it follows from Lemma 4.2 that $A=0$. Lemma 4.2 also shows that we may have $A=0$ when $N>3$. For later use notice that (4.23) shows that $w(\tilde{s}, \tilde{y}, \theta)=0$ if $\tilde{\xi}_{2}\left(\tilde{y}, \theta^{\prime}\right)=0$ for all $\theta^{\prime}$. Therefore if we denote by $V_{0}^{\prime \prime}$ the set of all $(s, y) \in V_{0}^{\prime}$ such that the integral curve of $X$ through $(s, y)$ intersects $H$ at some point $\left(s_{0}, y_{0}\right) \in J\left(\left\{t_{0}\right\} \times K_{1}\right)$, we obtain

$$
w_{2}(s, y, \theta)=0 \quad \text { if }(s, y) \notin V_{0}^{\prime \prime} \text {. }
$$

Since $\tilde{\zeta}_{2}(t, x, \theta)$ is periodic of period $2 \pi$ with respect to $\theta$, it follows from the uniqueness of the solutions of (4.15), (4.16) that $w_{2}(s, y, \theta)$ is also periodic of period $2 \pi$ with respect to $\theta$. If $s \in \mathbb{R}, y \in \mathbb{R}^{N}$, and if we have a function $\tau$ defined at $(s, y, \theta)$ for all $\theta \in[0,2 \pi]$, let us introduce the notation $\tau^{\#}(s, y)=$ $\int_{0}^{2 \pi} \tau(s, y, \theta) d \theta$. If we integrate $(4.15)$ with respect to $\theta$ over $[0,2 \pi]$, we obtain

$$
\left(2 X+\square^{\prime} \Phi\right) w_{2}^{\#}(s, y)=0 \quad \text { if }(s, y) \in V_{0}^{\prime}
$$


Since we assume that $\int_{0}^{2 \pi} \zeta_{2}(x, \theta) d \theta=0$ for all $x \in \mathbb{R}^{N}$, an integration of (4.16) with respect to $\theta$ over $[0,2 \pi]$ gives that

$$
w_{2}^{\#}(s, y)=0 \quad \text { if }(s, y) \in J\left(\left\{t_{0}\right\} \times\left(\Omega_{b} \backslash \bar{\Omega}_{a}\right)\right) .
$$

(4.25), (4.26) imply that $w_{2}^{\#}(s, y)=0$ if $(s, y) \in V_{0}^{\prime}$. Put $w_{2}(s, y, \theta)=0$ if $(s, y) \in \mathscr{D} \backslash V_{0}^{\prime}$ and $\theta \in \mathbb{R}$. From (4.24) it follows that $w_{2} \in C^{\infty}(\overline{\mathscr{D}} \times \mathbb{R})$; furthermore $w_{2}(s, y, \theta)$ is periodic of period $2 \pi$ with respect to $\theta, w_{2}^{\#}=0$ on $\mathscr{D}$, and so if

$$
\partial_{\theta} Z_{2}(s, y, \theta)=w_{2}(s, y, \theta) \quad \text { for }(s, y) \in \mathscr{D} \text { and } \theta \in \mathbb{R},
$$

then $Z_{2}(s, y, \theta)$ is periodic of period $2 \pi$ with respect to $\theta$ and $\partial_{\theta} Z_{2}$ solves $F_{1}(s, y, \theta)=0$ if $(s, y) \in \mathscr{D}$ and $\theta \in \mathbb{R}$, since $\square^{\prime} Z_{1}=0$ in $\mathscr{D}$. (4.27) does not determine $Z_{2}$ uniquely, but $Z_{2}$ will be determined by imposing $Z_{2}^{\#}$ when solving the equation $F_{2}(s, y, \theta)=0$ at the next step.

Solving $F_{k}(s, y, \theta)=0,2 \leq k \leq \mu-1$. For achieving that $F_{1}(s, y, \theta)=0$, we have determined $Z_{1}$ and $\partial_{\theta} Z_{2}$, with $Z_{1}(s, y)=0$ when $|y| \geq s+r$ and $\partial_{\theta} Z_{2}(s, y, \theta)=0$ if $(s, y) \in \mathscr{D} \backslash V_{0}^{\prime \prime}$, such that $\partial_{\theta} Z_{2}(s, y, \theta)$ is periodic of period $2 \pi$ with respect to $\theta$ and $\left(\partial_{\theta} Z_{2}\right)^{\#}=0$. We shall now show by induction on $k$ that

There exists a (unique) choice of functions $Z_{l}^{\#}(s, y) \in C^{\infty}(\overline{\mathscr{D}})$ and $\partial_{\theta} Z_{l+1}(s, y, \theta) \in C^{\infty}(\overline{\mathscr{D}} \times \mathbb{R}), 1 \leq l \leq k$, which satisfy the simultaneous conditions $F_{l}(s, y, \theta)=0,1 \leq l \leq k$, and the following conditions: (1) $Z_{l}^{\#}(s, y)=0$ when $|y| \geq s+r$;

(2) $\left(K_{0}+(N-1) s\right)^{j} Z_{l}^{\#}(s, y)=\left(s^{2}-|y|^{2}\right)^{-(N-1) / 2}\left(\tilde{\omega}_{l}^{j} \circ J\right)^{*}(s, y)$ if $j=0,1$ and $(s, y) \in H^{*}$; (3) $\partial_{\theta} Z_{l+1}(s, y, \theta)$ is periodic of period $2 \pi$ with respect to $\theta$, vanishes if $(s, y) \in$ $\mathscr{D} \backslash V_{0}^{\prime \prime}$, and satisfies $\left(\partial_{\theta} Z_{l+1}\right)^{\#}(s, y)=0$ if $(s, y) \in \mathscr{D} ;(4)$ $\partial_{\theta} Z_{l+1}(s, y, \theta)=\left(s^{2}-|y|^{2}\right)^{-(N-1) / 2} \tilde{\zeta}_{l+1}(J(s, y), \theta)$ if $(s, y) \in$ $J\left(\left\{t_{0}\right\} \times\left(\Omega_{b} \backslash \bar{\Omega}_{a}\right)\right)$ and $\theta \in \mathbb{R}$.

Remark 4.1. A sufficiently regular function $E(s, y, \theta)$ satisfies $\partial_{\theta} E=e_{1}$, $E^{\#}=e_{2}$ if and only if

$E(s, y, \theta)=\frac{1}{2 \pi} e_{2}(s, y)+\int_{0}^{\theta} e_{1}\left(s, y, \theta^{\prime}\right) d \theta^{\prime}+\frac{1}{2 \pi} \int_{0}^{2 \pi}\left(\theta^{\prime}-2 \pi\right) e_{1}\left(s, y, \theta^{\prime}\right) d \theta^{\prime}$.

If we apply (4.29) with $e_{1}=\partial_{\theta} Z_{l}$ and $e_{2}=Z_{l}^{\#}, 1 \leq l \leq k$, it follows from (4.28) that the functions $Z_{l}, 1 \leq l \leq k$, given by (4.29), are determined when the conditions $F_{l}(s, y, \theta)=0,1 \leq l \leq k$, have been satisfied by the choice of $Z_{l}^{\#}, \partial_{\theta} Z_{l+1}, 1 \leq l \leq k$, as indicated in (4.28). Moreover, if $1 \leq l \leq k$, $Z_{l} \in C^{\infty}(\overline{\mathscr{D}} \times \mathbb{R}), Z_{l}(s, y, \theta)$ is periodic of period $2 \pi$ with respect to $\theta$ and independent of $\theta$ if $(s, y) \notin V_{0}^{\prime \prime}$. Now it is easily checked that $|y| \leq s+r$ if $(s, y) \in V_{0}^{\prime \prime}$, so (4.28) and (4.29) imply that $Z_{l}(s, y, \theta)=\partial_{\theta} Z_{l+1}(s, y, \theta)=0$ if $1 \leq l \leq k$ and $|y| \geq s+r$.

(4.28) has already been checked when $k=1$; so assume that it has been proved if $k \leq n-1 \quad(n<\mu)$ and let us show that it still holds if $k=n$. To simplify notations in (4.7), it is convenient to put

$$
f_{k}(s, y, \theta)=\mathscr{F}_{k}\left(s, y,\left(\partial_{\theta}^{l} D^{\alpha} Z_{j}(s, y, \theta)\right)_{l+|\alpha| \leq 2,1 \leq j \leq k(l, \alpha)}\right),
$$


$g_{k}=\square^{\prime} Z_{k}-Q Z_{k} \cdot \partial_{\theta}^{2} Z_{2}+f_{k}$. We first solve the simultaneous equations $F_{l}(s, y, \theta)=0$ for $1 \leq l \leq n-1$, using (4.28) with $k=n-1$. From Remark 4.1 it follows that

$$
\partial_{\theta} g_{n}(s, y, \theta)=0 \quad \text { if }(s, y) \in \mathscr{D} \backslash V_{0}^{\prime \prime} .
$$

Since we want both $Z_{2}(s, y, \theta)$ and $Z_{n+1}(s, y, \theta)$ to be periodic of period $2 \pi$ with respect to $\theta$, it follows from (4.7) that we should have $F_{n}^{\#}=g_{n}^{\#}$; hence for satisfying $F_{n}(s, y, \theta)=0$ with a function $Z_{n+1}(s, y, \theta)$ periodic of period $2 \pi$ with respect to $\theta$, a necessary condition is that

$$
g_{n}^{\#}(s, y)=0 \quad \text { if }(s, y) \in \mathscr{D} .
$$

Now we may write $\left(Q Z_{n} \cdot \partial_{\theta}^{2} Z_{2}\right)^{\#}=\mathscr{I}_{n}+\mathscr{J}_{n}$, where $\mathscr{I}_{n}=\left(\partial_{\theta}\left(Q Z_{n} \cdot \partial_{\theta} Z_{2}\right)\right)^{\#}$ and $\mathscr{J}_{n}=-\left(Q \partial_{\theta} Z_{n} \cdot \partial_{\theta} Z_{2}\right)^{\#}$. Since $\partial_{\theta} Z_{2}(s, y, 0)=\partial_{\theta} Z_{2}(s, y, 2 \pi)$, we have $\mathscr{I}_{n}(s, y)=Q\left(\partial_{\theta} Z_{n}\right)^{\#}(s, y) \cdot \partial_{\theta} Z_{2}(s, y, 0)$. Now because of (4.28) with $k=$ $n-1$, we have $\left(\partial_{\theta} Z_{n}\right)^{\#}=0$, so $\mathscr{I}_{n}=0$. Hence if we put $h_{n}=\mathscr{I}_{n}-f_{n}^{\#},(4.31)$ can be written

$$
\square^{\prime} Z_{n}^{\#}(s, y)=h_{n}(s, y) \quad \text { if }(s, y) \in \mathscr{D} .
$$

Using (4.28) with $k=n-1$, we have solved the equations $F_{l}(s, y, \theta)=0$, $1 \leq l \leq n-1$; it follows from Remark 4.1 that $h_{n}$ is determined and that $h_{n}(s, y)=0$ if $|y| \geq s+r$. Now let $Y(s, y)$ be the solution of the following Cauchy problem:

$$
\begin{gathered}
\square^{\prime} Y(s, y)=h_{n}(s, y) \quad \text { if }(s, y) \in \mathscr{D}, \\
\left(K_{0}+(N-1) s\right)^{j} Y(s, y)=\left(s^{2}-|y|^{2}\right)^{-(N-1) / 2}\left(\tilde{\omega}_{n}^{j} \circ J\right)^{*}(s, y) \\
\text { if } j=0,1 \text { and }(s, y) \in H^{*} .
\end{gathered}
$$

Then $Y \in C^{\infty}(\overline{\mathscr{D}})$ and $Y(s, y)=0$ if $|y| \geq s+r$. We choose $Z_{n}^{\#}=Y$; then of course (4.32) holds. Since $\partial_{\theta} Z_{n} \in C^{\infty}(\overline{\mathscr{D}} \times \mathbb{R})$ has already been chosen for solving the equation $F_{n-1}(s, y, \theta)=0$ (cf. (4.28)), it follows from (4.29) (with $e_{1}=\partial_{\theta} Z_{n}$ and $\left.e_{2}=Z_{n}^{\#}\right)$ that $Z_{n} \in C^{\infty}(\overline{\mathscr{D}} \times \mathbb{R})$ is determined now and that $Z_{n}(s, y, \theta)$ is periodic of period $2 \pi$ with respect to $\theta$. Because of (4.28) with $k=n-1$ and of Remark 4.1, all $Z_{j}(s, y, \theta), j<n$, are also periodic of period $2 \pi$ with respect to $\theta$; therefore we reach the conclusion that $g_{n}$ is determined now and that $g_{n}(s, y, \theta)$ is also periodic of period $2 \pi$ with respect to $\theta$. Now consider the Cauchy problem

$$
\begin{gathered}
\left(2 X+\square^{\prime} \Phi-\left(Q Z_{1}+A \partial_{\theta} Z_{2}\right) \partial_{\theta}-A \partial_{\theta}^{2} Z_{2}\right) w_{n+1}(s, y, \theta) \\
=-g_{n}(s, y, \theta) \quad \text { if }(s, y) \in V_{0}^{\prime}, \theta \in \mathbb{R}, \\
w_{n+1}(s, y, \theta)=\left(s^{2}-|y|^{2}\right)^{-(N-1) / 2} \tilde{\zeta}_{n+1}(J(s, y), \theta) \\
\quad \text { if }(s, y) \in J\left(\left\{t_{0}\right\} \times\left(\Omega_{b} \backslash \bar{\Omega}_{a}\right)\right), \theta \in \mathbb{R} .
\end{gathered}
$$

From (4.30), (4.32), and (4.29) with $e_{1}=\partial_{\theta} g_{n}$ and $e_{2}=g_{n}^{*}=0$, it follows that $g_{n}(s, y, \theta)=0$ if $(s, y) \in \mathscr{D} \backslash V_{0}^{\prime \prime}$. We may solve the linear problem (4.35), (4.36) by straightening out the integral curves of $X$, using arguments similar to those used for solving (4.15), (4.16). It follows that (4.35), (4.36) has a unique $C^{\infty}\left(\overline{V_{0}^{\prime}} \times \mathbb{R}\right)$ solution $w_{n+1}$, such that $w_{n+1}(s, y, \theta)=0$ if $(s, y) \in V_{0}^{\prime} \backslash V_{0}^{\prime \prime}$. 
Since $g_{n}(s, y, \theta)$ is periodic of period $2 \pi$ with respect to $\theta$, it follows from the uniqueness of the solutions of (4.35), (4.36) that $w_{n+1}(s, y, \theta)$ is also periodic of period $2 \pi$ with respect to $\theta$. Furthermore, integrating (4.35) and (4.36) with respect to $\theta$ over $[0,2 \pi]$, and using (4.31) and the assumption that $\int_{0}^{2 \pi} \zeta_{n+1}(x, \theta) d \theta=0$ for all $x \in \mathbb{R}^{N}$, we obtain that (4.25), (4.26) still hold with $w_{2}^{\#}$ replaced by $w_{n+1}^{\#}$. Therefore $w_{n+1}^{\#}(s, y, \theta)=0$ if $(s, y) \in V_{0}^{\prime}$. Put $w_{n+1}(s, y, \theta)=0$ if $(s, y) \in \mathscr{D} \backslash V_{0}^{\prime}$. Then $w_{n+1} \in C^{\infty}(\overline{\mathscr{D}} \times \mathbb{R}), w_{n+1}(s, y, \theta)$ is periodic of period $2 \pi$ with respect to $\theta$, and $w_{n+1}^{\#}=0$ on $\mathscr{D}$. So if

$$
\partial_{\theta} Z_{n+1}(s, y, \theta)=w_{n+1}(s, y, \theta) \quad \text { if }(s, y) \in \mathscr{D}, \theta \in \mathbb{R},
$$

then the condition $F_{n}(s, y, \theta)=0$ is satisfied if $(s, y) \in \mathscr{D}, \theta \in \mathbb{R}$. (4.28) is now completely verified for $k=n$. Notice that if $Z_{n+1}$ satisfies (4.37), then $Z_{n+1}(s, y, \theta)$ is periodic of period $2 \pi$ with respect to $\theta$.

Summing up, the conditions $F_{j}(s, y, \theta)=0,1 \leq j \leq \mu-1$, can be satisfied by a suitable choice of $Z_{k}, 1 \leq k \leq \mu-1$, and of $\partial_{\theta} Z_{\mu}$ (with the properties described in (4.28) and the consequences given in Remark 4.1). To determine $Z_{\mu}$ by means of (4.29) we must still choose $Z_{\mu}^{\#} ; Z_{\mu}^{\#}$ should also satisfy suitable initial conditions on $H$. For any choice of $Z_{\mu}^{\#} \in C^{\infty}(\overline{\mathscr{D}})$, the corresponding $Z^{\varepsilon}$ would be an approximate solution of (3.1); but some caution is necessary if we want to obtain from $Z^{\varepsilon}$ a smooth approximate solution of $(2.1)$ when $t>t_{0}$ and $x \in \mathbb{R}^{N}$. We revert the transformation $z \mapsto Z$ used for getting (3.1); namely we put (for $1 \leq j \leq \mu-1$, and later on also for $j=\mu$ ) (4.38)

$$
z_{j}(t, x, \theta)=\left(t^{2}-|x|^{2}\right)^{-(N-1) / 2} Z_{j}(J(t, x), \theta) \quad \text { if } t>t_{0} \text { and }(t, x) \in C_{+} ;
$$

and we also put

$$
z_{j}(t, x, \theta)=0 \quad \text { if } t>t_{0} \text { and }(t, x) \notin C_{+} .
$$

Assume first that $1 \leq j \leq \mu-1$. Since, by construction, we have $Z_{j}(s, y, \theta)=$ 0 for $(s, y) \in \mathscr{D}$ and $|y| \geq s+r$, where $r=\left(t_{0}-M\right)^{-1}$, it follows from (4.38) that $z_{j}(t, x, \theta)=0$ if $|x| \geq t+M-t_{0}, t>t_{0}$, and $(t, x) \in C_{+}$. Hence $z_{j} \in C^{\infty}\left(\left[t_{0},+\infty\left[\times \mathbb{R}^{N} \times \mathbb{R}\right)\right.\right.$ if $1 \leq j \leq \mu-1$. To obtain the same property for $z_{\mu}$, choose $Z_{\mu}^{\#} \in C^{\infty}(\overline{\mathscr{D}})$, vanishing for $|y| \geq s+r$; besides take $Z_{\mu}^{\#}$ satisfying the initial conditions

$$
\begin{array}{r}
\left(K_{0}+(N-1) s\right)^{i} Z_{\mu}^{\#}(s, y)=\left(s^{2}-|y|^{2}\right)^{-(N-1) / 2}\left(\tilde{\omega}_{\mu}^{i} \circ J\right)(s, y) \\
\text { if } i=0,1 \text { and }(s, y) \in H .
\end{array}
$$

Define $Z_{\mu}$ by means of (4.29), and then $z_{\mu}$ by the relations (4.38), (4.39) (with $j=\mu$ ). Repeating the reasoning made for $z_{j}, j<\mu$, we obtain that $z_{\mu} \in C^{\infty}\left(\left[t_{0},+\infty\left[\times \mathbb{R}^{N} \times \mathbb{R}\right)\right.\right.$. (The same would also hold e.g. if $Z_{\mu}^{\#}(s, y)=0$ only when $|y| \geq s+r^{\prime}$ with some $r^{\prime}>r$ ). Because of (4.38), (4.39), the functions $z_{j}(t, x, \theta), 1 \leq j \leq \mu$, are independent of $\theta$ when $J(t, x) \notin V_{0}^{\prime \prime}$, that is, when $(t, x) \notin W$. Notice that it follows from the construction of $Z_{1}, \ldots, Z_{\mu}$ that $Z^{\varepsilon} \in C^{\infty}(\overline{\mathscr{D}})$, that $Z^{\varepsilon}(s, y)=0$ if $|y| \geq s+r$, and that for each $\varepsilon_{0}>0$, one can find $C_{\alpha} \in \mathbb{R}$ such that

$$
\left|D^{\alpha} Z^{\varepsilon}(s, y)\right| \leq C_{\alpha}\left(\varepsilon+\varepsilon^{2-|\alpha|}\right) \quad \text { if }(s, y) \in \mathscr{D} \text { and } 0<\varepsilon \leq \varepsilon_{0} .
$$

We may now complete the proof of Theorem 1. Assume first that $t_{0}>0$ is so large that all the steps of the construction of $Z^{\varepsilon}$ and all the estimates of 
$Z^{\varepsilon}$ described above are valid. Then let $Z^{\varepsilon}, R_{\mu}$ be as above. Define $z_{j}$, $1 \leq j \leq \mu$ as in (4.38), (4.39); put $z^{\varepsilon}(t, x)=\left(t^{2}-|x|^{2}\right)^{-(N-1) / 2} Z^{\varepsilon}(J(t, x))$ if $t \geq t_{0}$ and $(t, x) \in C_{+}, z^{\varepsilon}(t, x)=0$ if $t \geq t_{0}$ and $(t, x) \notin C_{+}$; define also $R^{\varepsilon}(t, x)=\left(t^{2}-|x|^{2}\right)^{-(N+3) / 2} R_{\mu}(J(t, x), \varphi(t, x) / \varepsilon, \varepsilon)$ if $t \geq t_{0}$ and $(t, x) \in C_{+}, R^{\varepsilon}(t, x)=0$ if $t \geq t_{0}$ and $(t, x) \notin C_{+}$. All the properties announced in Theorem 1 follow easily if we make use of (4.41), (4.10) (and of Remark 2.5). Now if $t_{0}$ is any real number, Theorem 1 also follows because we may, by a translation of $t$, reduce to the case where $t_{0}>0$ is as large as we please. The proof of Theorem 1 is complete.

Remark 4.2. One could have tried to construct $z^{\varepsilon}(t, x)$ directly, without using the Kelvin transform, by writing down conditions of the type $F_{k}=0$ coming from (2.1) (and not from (3.1) anymore), and using the given initial data. Actually one can check that this yields the same $z_{j}, 1 \leq j<\mu$, and the same $\partial_{\theta} z_{\mu}$ as we have obtained. To see this, put for simplicity $\mathscr{P}(z)=$ $\square z-\sum_{0 \leq i, j \leq N} f^{i j}\left(z, z^{\prime}\right) \partial_{i j}^{2} z-f\left(z, z^{\prime}\right)$. Assume that $t_{0}$ is large and that $z_{j}$, $1 \leq j \leq \mu$, are defined by (4.38), (4.39). Denote by $\varphi$ a $C^{\infty}\left(\left[t_{0},+\infty\left[\times \mathbb{R}^{N}\right)\right.\right.$ extension of the function $\varphi \in C^{\infty}(\bar{V})$ defined before the statement of Theorem 1. If $\lambda \in \mathbb{R}$, we may argue as for (4.9), and write

$$
\begin{aligned}
& \mathscr{P}\left(\sum_{1 \leq j \leq \mu} \varepsilon^{j} z_{j}\left(t, x, \frac{\varphi+\lambda}{\varepsilon}\right)\right) \\
& \quad=\sum_{1 \leq k \leq \mu-1} \varepsilon^{k} \bar{F}_{k}\left(t, x, \frac{\varphi+\lambda}{\varepsilon}\right)+\varepsilon^{\mu} \bar{R}_{\mu}\left(t, x, \frac{\varphi+\lambda}{\varepsilon}, \varepsilon\right),
\end{aligned}
$$

where $\bar{F}_{k}, \bar{R}_{\mu}$ are constructed with (2.1) in the same way as $F_{k}, R_{\mu}$ were constructed with (3.1); so to pass from (4.6), (4.7) to the definition of the functions $\bar{F}_{k}$, one should replace $s, y, g^{i j}, g, Z_{j}, \Phi$ by $t, x, f^{i j}, f$, $z_{j}, \varphi+\lambda$. Now (4.5) shows that the functions $F_{k}(s, y, \theta), R_{\mu}(s, y, \theta, \varepsilon)$ are unchanged if $\Phi$ is replaced by $\Phi+\lambda$ since only derivatives of order 1 and 2 of the phase (and not the phase itself) appear there. Hence from the definition (4.38), (4.39) of $z_{j}$, it follows that the left-hand side of (4.42) is bounded by $C \varepsilon^{\mu}$ as $\varepsilon \rightarrow 0$, where $C$ is a constant independent of $\lambda, \varepsilon$ (and of $t, x)$; hence the right-hand side of (4.42) is also bounded by $C \varepsilon^{\mu}$ as $\varepsilon \rightarrow 0$. Now a formula analogous to (4.5) shows that the functions $\bar{F}_{k}(t, x, \theta)$, $\bar{R}_{\mu}(t, x, \theta, \varepsilon)$ appearing in (4.42) are independent of $\lambda$. Therefore if we now take $t_{1}>t_{0}, x_{1} \in \mathbb{R}^{N}, \theta_{1} \in \mathbb{R}, t=t_{1}, x=x_{1}, \lambda=\varepsilon \theta_{1}-\varphi\left(t_{1}, x_{1}\right)$, we obtain from (4.42) that $\bar{F}_{k}\left(t_{1}, x_{1}, \theta_{1}\right)=0,1 \leq k \leq \mu-1$. (Of course, the introduction of $\lambda$ is not necessary to obtain that result when $\left(t_{1}, x_{1}\right)$ does not belong to the set $W$ occurring in Theorem 1(i)). Since $t_{1}, x_{1}, \theta_{1}$ are arbitrary, we conclude that if $z_{j}, 1 \leq j \leq \mu$, are defined by (4.38), (4.39), we obtain that $\bar{F}_{k}(t, x, \theta)=0$ if $1 \leq k \leq \mu-1, t>t_{0}, x \in \mathbb{R}^{N}, \theta \in \mathbb{R}$. Now by the same arguments as those used for proving (4.28), we see that there is only one way of constructing $z_{l}(t, x, \theta), 1 \leq l \leq \mu-1$, and $\partial_{\theta} z_{\mu}(t, x, \theta)$, all periodic of period $2 \pi$ with respect to $\theta$, with $z_{1}$ independent of $\theta$, such that the mapping $\theta \mapsto \partial_{\theta} z_{\mu}(t, x, \theta)$ has an antiderivative periodic of period $2 \pi$, and such that the following conditions hold: $\bar{F}_{k}(t, x, \theta)=0$ if $1 \leq k \leq \mu-1, t>t_{0}$, $x \in \mathbb{R}^{N}, \theta \in \mathbb{R}, \partial_{\theta} z_{l}(t, x, \theta)=\zeta_{l}(x, \theta)$ and $\partial_{0}^{j}\left(\int_{0}^{2 \pi} z_{n}(t, x, \theta) d \theta\right)=\omega_{n}^{j}(x)$ 
if $2 \leq l \leq \mu, j=0,1,1 \leq n \leq \mu-1, t=t_{0}, x \in \mathbb{R}^{N}, \theta \in \mathbb{R}$. Hence the functions $z_{j}, 1 \leq j \leq \mu-1$, and $\partial_{\theta} z_{\mu}$ defined by means of (4.38), (4.39) when $t_{0}$ is large are the same as those one would have obtained by solving the equations $\bar{F}_{k}=0,1 \leq k \leq \mu-1$, taking the initial conditions given by $\zeta_{l}, \omega_{n}^{j}$ into account. However, working with $Z^{\varepsilon}$ gives immediately precise decay estimates for $z^{\varepsilon}, R^{\varepsilon}$; considering approximate solutions defined beyond $\mathscr{D} \cap C_{-}$will also be useful for proving Theorem 2 in the next section.

Remark 4.3. The strong convexity assumption associated to $\psi$ is important. First it prevents characteristic surfaces from having caustics (but for this mere convexity would be enough), but it also plays a role in the global existence of the function $z_{2}(t, x, \theta)$; for this global existence, the size of $N$ also matters. To see this, let us keep all the assumptions and notations introduced in $\S 2$, except that now we do not assume any more that the sets $\Omega_{\lambda}$ are strongly convex, but only that they are convex (so no caustics appear). Assume that $\varphi$ is a solution of $\partial_{0} \varphi+\left|\partial_{x} \varphi\right|=0$ in $V$ such that $\left.\varphi\right|_{t=t_{0}}=\psi$, and let us try to find an approximate solution $z^{\varepsilon}(t, x)=\varepsilon z_{1}(t, x)+\sum_{2 \leq j \leq \mu} \varepsilon^{j} z_{j}(t, x, \varphi(t, x) / \varepsilon)$ of (2.1) with $z_{j}$ satisfying initial conditions when $t=t_{0}$ as described in the statement of Theorem 1. Let us apply the method used above, but directly in the variables $(t, x)$ (cf. Remark 4.2). Assume that $f^{i j}(z, p)=b^{i j} z+$ $\sum_{0 \leq k \leq N} b^{i j k} p_{k}+\mathscr{O}\left(z^{2}+|p|^{2}\right)$ as $(z, p) \rightarrow(0,0)$, with $b^{i j}, b^{i j k} \in \mathbb{R}$. Observe that $\partial_{0} \varphi$ never vanishes in $V$; indeed if $m \in \Omega_{b} \backslash \bar{\Omega}_{a}$ and

$$
h(t, m)=\left(t, m+\left(t-t_{0}\right)\left|\psi^{\prime}(m)\right|^{-1} \psi^{\prime}(m)\right)
$$

as before, it follows from the classical Hamilton-Jacobi theory that $\partial_{0} \varphi(h(t, m))$ $=-\left|\psi^{\prime}(m)\right|$ for all $t>t_{0}$ (cf. e.g. [3]). Therefore we may define

$$
\begin{aligned}
& A_{1}=\frac{1}{2 \partial_{0} \varphi} \sum_{0 \leq i, j, k \leq N} b^{i j k} \partial_{i} \varphi \cdot \partial_{j} \varphi \cdot \partial_{k} \varphi, \\
& B_{1}=\frac{1}{2 \partial_{0} \varphi} \sum_{0 \leq i, j \leq N} \partial_{i} \varphi \cdot \partial_{j} \varphi \cdot\left(b^{i j}+\sum_{0 \leq k \leq N} b^{i j k} \partial_{k}\right) z_{1}, \\
& X_{1}=\partial_{0}-\sum_{1 \leq j \leq N} \frac{\partial_{j} \varphi}{\partial_{0} \varphi} \partial_{j} .
\end{aligned}
$$

When $(t, x) \in V$, the condition $\bar{F}_{1}=0$ originating from (2.1) (see (4.42)) reads (cf. (4.6))

$$
\begin{array}{r}
2 \partial_{0} \varphi(t, x)\left(X_{1}+\frac{\square \varphi}{2 \partial_{0} \varphi}-\left(B_{1}+A_{1} \partial_{\theta} z_{2}\right) \partial_{\theta}\right) \partial_{\theta} z_{2}(t, x, \theta)+\square z_{1}(t, x)=0 \\
\text { if }(t, x) \in V, \theta \in \mathbb{R} .
\end{array}
$$

If $m \in \Omega_{b} \backslash \bar{\Omega}_{a}$, let $\lambda_{1}(m) \leq \lambda_{2}(m) \leq \cdots \leq \lambda_{N-1}(m)$ be the principal curvatures of $\partial \Omega_{\psi(m)}$ at $m$ in the direction of $-\left|\psi^{\prime}(m)\right|^{-1} \psi^{\prime}(m)$. Since $\Omega_{\psi(m)}$ is convex, we have $\lambda_{1}(m) \geq 0$. Put

$$
\mathscr{L}(t, m)=\frac{1}{2} \sum_{1 \leq j \leq N-1} \frac{\lambda_{j}(m)}{1+\lambda_{j}(m)\left(t-t_{0}\right)} .
$$


Now the Hamilton-Jacobi theory shows that $\varphi(h(t, m))=\psi(m)$; therefore a simple computation based on inverting the map $m \mapsto h(t, m)$ shows that $\left(\square \varphi / 2 \partial_{0} \varphi\right)(h(t, m))=\mathscr{L}(t, m)$ (cf. also Lemma 3.1 of [3]). The HamiltonJacobi theory also implies that $\partial_{i} \varphi(h(t, m))=\partial_{i} \psi(m)$ if $i>0$ and that (cf. above) $\partial_{0} \varphi(h(t, m))=-\left|\psi^{\prime}(m)\right|$. It follows that $A_{1}(h(t, m))$ is independent of $t$, so let us write $\widetilde{A_{1}}(m)=A_{1}(h(t, m))$. Put $B_{2}(t, m)=-B_{1}(h(t, m))$. For fulfilling (4.43) we must choose $z_{1}$ such that $\square z_{1}(t, x)=0$ if $t>t_{0}$, $x \in \mathbb{R}^{N}$ (cf. (4.13)). We should also have $\partial_{0}^{j} z_{1}\left(t_{0}, x\right)=\omega_{1}^{j}(x)$ if $j=0,1$ and $x \in \mathbb{R}^{N}$. Since (4.43) holds and $\square z_{1}=0$, the equation (corresponding to (4.19)) for $\tilde{w}_{2}(t, m, \theta) \equiv \partial_{\theta} z_{2}(h(t, m), \theta)$ is

$$
\left(\partial_{0}+\mathscr{L}+\left(B_{2}-\tilde{A}_{1} \tilde{w}_{2}\right) \partial_{\theta}\right) \tilde{w}_{2}(t, m, \theta)=0 \quad \text { if } t>t_{0}, m \in \Omega_{b} \backslash \bar{\Omega}_{a}, \theta \in \mathbb{R} .
$$

Put $w(t, m, \theta)=\exp \left(\int_{t_{0}}^{t} \mathscr{L}(s, m) d s\right) \tilde{w}_{2}(t, m, \theta)$ and

$$
A_{2}(t, m)=-\tilde{A_{1}}(m) \exp \left(-\int_{t_{0}}^{t} \mathscr{L}(s, m) d s\right) .
$$

Then (4.44) gives

$$
\left(\partial_{0}+\left(B_{2}+A_{2} w\right) \partial_{\theta}\right) w(t, m, \theta)=0 \quad \text { if } t>t_{0}, m \in \Omega_{b} \backslash \bar{\Omega}_{a}, \theta \in \mathbb{R} .
$$

Furthermore if we want to fulfill the initial condition $\partial_{\theta} z_{2}\left(t_{0}, x, \theta\right)=\zeta_{2}(x, \theta)$, we should have

$$
w\left(t_{0}, m, \theta\right)=\zeta_{2}(m, \theta) \quad \text { if } m \in \Omega_{b} \backslash \bar{\Omega}_{a}, \theta \in \mathbb{R} .
$$

Assume that for some $n \in \mathbb{N}$ with $n \leq N-1$, we have $\lambda_{j}(m)>0$ if and only if $j \geq N-n$ (if $n=0$, we mean by this that no $\lambda_{j}(m)$ is $>0$ ). In the strongly convex case treated in Theorem 1, we have $n=N-1$, but assume now that $n \leq 2$ (and $N$ is arbitrary). Also assume that $\tilde{A}_{1}(m) \neq 0$. For some $\tau>0$, we have $\mathscr{L}(t, m) \leq n / 2\left(t-t_{0}+\tau\right)$ if $t>t_{0}$; hence it follows at once that

$$
\left|\int_{t_{0}}^{t} A_{2}(s, m) d s\right| \rightarrow+\infty \quad \text { as } t \rightarrow+\infty .
$$

But $(1+|t|+|x|)^{(N-1) / 2}\left|\partial^{\alpha} z_{1}(t, x)\right|$ is bounded (cf. e.g. [7]); hence if $N>3$ (or if e.g. $z_{1} \equiv 0$ and $N \leq 3$ ), we obtain that, for some $C>0$,

$$
\left|\int_{t_{0}}^{t} B_{2}(s, m) d s\right| \leq C \quad \text { if } t>t_{0}, m \in \Omega_{b} \backslash \bar{\Omega}_{a} .
$$

Now let us try to solve (4.45), (4.46). If (4.47), (4.48) hold, it easily follows from the method of characteristics that even if we impose that $\sup \left|\partial_{\theta} \zeta_{2}\right| \leq \delta$, with $\delta$ as small as we please, suitable choices of $\zeta_{2}$ will produce intersecting characteristics (for (4.45)) on each of which $w$ should take a different constant value; therefore (4.45), (4.46) will have no global smooth solution for such choices of $\zeta_{2}$.

\section{Proof of Theorem 2}

Let us keep the notations of the preceding sections. Write

$$
\mathscr{D}^{*}=\left\{(s, y) \in \mathbb{R} \times \mathbb{R}^{N},-L(y)<s<0\right\} .
$$


To prove Theorem 2, the main point will be to construct, for $\varepsilon$ small, a solution $\underline{Z}^{\varepsilon}$ of $(3.1)$ in $\mathscr{D}^{*} \cap C_{-}$such that $D_{0}^{k}\left(\underline{Z}^{\varepsilon}-Z^{\varepsilon}\right)=0$ on $H^{*}$ when $k=0,1$. In order to use the results of [5], we shall truncate $g^{i j}, g$, and then reduce to a symmetrizable hyperbolic first order system in a standard way as e.g. in [4]. Let $\left.\delta_{1} \in\right] 0,1\left[\right.$ be a constant to be chosen later. Let $\hat{g}^{i j}(s, y, Z, P), 0 \leq i$, $j \leq N$, and $\hat{g}(s, y, Z, P)$ be $C^{\infty}\left(\mathbb{R} \times \mathbb{R}^{N} \times \mathbb{R} \times \mathbb{R}^{N+1}\right)$ functions satisfying the following conditions: (1) $\hat{g}^{i j}=\hat{g}^{j i}$; (2) $\hat{g}^{i j}=g^{i j}$ in a neighbourhood $\mathscr{V}$ of $\left\{(s, y, 0,0),(s, y) \in \bar{C}_{-}\right\} ;(3) \hat{g}=g$ in $\mathscr{V} ;(4) \hat{g}^{i j}(s, y, 0,0)=$ $\partial_{Z P}^{\alpha} \hat{g}(s, y, 0,0)=0$ if $(s, y) \in \mathbb{R} \times \mathbb{R}^{N}$ and $|\alpha| \leq 1$. Choose $\mathscr{V}$ and $\hat{g}^{i j}, \hat{g}$ in such a way that for some $r_{3}>0, \sum_{0 \leq i, j \leq N}\left|\hat{g}^{i j}(s, y, Z, P)\right|+$ $|\hat{g}(s, y, Z, P)|=0$ if $(s, y) \in \mathscr{D}^{*},|y| \geq r_{3}, Z \in \mathbb{R}$, and $P \in \mathbb{R}^{N+1}$, and that $\sum_{0<i, j \leq N}\left|\hat{g}^{i j}(s, y, Z, P)\right| \leq \delta_{1}$ if $(s, y) \in \mathscr{D}^{*}, Z \in \mathbb{R}$, and $P \in \mathbb{R}^{N+1}$. We shall replace the study of (3.1) by the study of

$$
\square^{\prime} Z=\sum_{0 \leq i, j \leq N} \hat{g}^{i j}(s, y, Z, D Z) D_{i j}^{2} Z+\hat{g}(s, y, Z, D Z) .
$$

This is justified since, in $\mathscr{D} \cap C_{-}$, the solutions $Z$ we shall handle will be small and will have a small gradient. Dividing $(5.1)$ by $1-\hat{g}^{00}(s, y, Z, D Z)$, we shall also assume that $\hat{g}^{00}=0$. Put $h^{i j}=\hat{g}^{i j}$ if $i, j>0, h^{i 0}=\hat{g}^{0 i}+\hat{g}^{i 0}$ if $i>0, u_{j}=D_{j} Z$ if $0 \leq j \leq N$. We have

$$
D_{0} Z-u_{0}=0, \quad D_{0} u_{i}-D_{i} u_{0}=0 \quad \text { if } 1 \leq i \leq N .
$$

If we put $U=\operatorname{tr}\left(Z, u_{0}, u_{1}, \ldots, u_{N}\right)$ (where ${ }^{\operatorname{tr}}$ means transpose), we can rewrite (5.1), (5.2) in the form

$$
D_{0} U+\sum_{1 \leq j \leq N} A_{j}(s, y, U) D_{j} U=F(s, y, U) .
$$

Here $A_{j}(s, y, U)$ is the $(N+2) \times(N+2)$ matrix with entries

$$
\left(A_{j}^{p, q}(s, y, U)\right)_{1 \leq p, q \leq N+2}
$$

such that $A_{j}^{2, q}=-\left(h^{j, q-2}+\delta^{j, q-2}\right)$ if $2 \leq q \leq N+2$ (where $\delta^{p, q}=1$ if $p=q$ and $\delta^{p, q}=0$ otherwise), $A_{j}^{j+2,2}=-1, A_{j}^{p, q}=0$ otherwise. $F(s, y, U)$ is the $(N+2) \times 1$ matrix $\operatorname{tr}\left(u_{0}, \hat{g}(s, y, U), 0, \ldots, 0\right)$. Let $S(s, y, U)$ be the $(N+2) \times(N+2)$ matrix with entries $\left(S^{p, q}(s, y, U)\right)_{1 \leq p, q \leq N+2}$, where $S^{1,1}=S^{2,2}=1, S^{i, j}=h^{i-2, j-2}+\delta^{i-2, j-2}$ if $3 \leq i, j \leq N+2, S^{p, q}=0$ otherwise. Put $\mathscr{B}=\left\{(s, y, U) \in \mathbb{R} \times \mathbb{R}^{N} \times \mathbb{R}^{N+2},(s, y) \in \overline{\mathscr{D}^{*}}\right\}$. It is not difficult to check the following properties:

(i) $S A_{j}(s, y, U)$ is symmetric if $1 \leq j \leq N$ and $(s, y, U)$ $\in \mathscr{B}$

(ii) there exists $C_{1}>0$ such that

$$
\sum_{1 \leq p, q \leq N+2}\left(\left|S^{p, q}(s, y, U)\right|+\sum_{1 \leq j \leq N}\left|A_{j}^{p, q}(s, y, U)\right|\right) \leq C_{1}
$$

for all $(s, y, U) \in \mathscr{B}$;

(iii) if $\delta_{1}$ is small enough, $S(s, y, U)$ is symmetric positive definite for all $(s, y, U) \in \mathscr{B}$ and there exists $C_{2}>0$ such that $S(s, y, U) \geq C_{2} I$ for all $(s, y, U) \in \mathscr{B}$, where $I$ is the identity $(N+2) \times(N+2)$ matrix. 
Let $Z^{\varepsilon}$ be the approximate solution of (3.1), of the form (4.2), constructed in the proof of Theorem 1 by solving (4.13), (4.14), (4.15), (4.16), (4.33), (4.34), (4.35), (4.36) and choosing $Z_{\mu}^{\#}$ satisfying (4.40) and vanishing if $|y| \geq s+r$. Keeping the same phase $\Phi$, replacing in (4.15), (4.33), (4.35) $g^{i j}$ by $\hat{g}^{i j}$ and $g$ by $\hat{g}$, and keeping (4.13), the initial conditions (4.14), (4.16), (4.34), (4.36), and the same $Z_{\mu}^{\#}$, we obtain in the same way an approximate solution $\widehat{Z}^{\varepsilon}$ of (5.1). Notice the following facts, whose verification is straightforward:

(1) the conditions $F_{k}=0$ are unchanged in $\mathscr{D} \cap C_{-}$if $g^{i j}, g$ are replaced by $\hat{g}^{i j}, \hat{g}$;

(2) if a (sufficiently regular) function $Z(s, y)$ satisfies $\square^{\prime} Z=0$ in $\mathscr{D} \cap C_{-}$ and $\left(K_{0}+(N-1) s\right)^{j} Z=0$ on $H^{*} \cap C_{-}$for $j=0,1$, then $Z=0$ in $\mathscr{D} \cap C_{-}$, so that a solution of (4.33), (4.34) is not modified in $\mathscr{D} \cap C_{-}$if $h_{n}$ is modified only outside $\mathscr{D} \cap C_{-}$;

(3) $V_{0}^{\prime} \cap C_{-}$is a union of intervals of integral curves of $X$, with one endpoint in $J\left(\left\{t_{0}\right\} \times\left(\Omega_{b} \backslash \bar{\Omega}_{a}\right)\right)$ and the other one in the boundary of $C_{-}$, so that a solution of (4.35), (4.36) is not modified in $\left(V_{0}^{\prime} \cap C_{-}\right) \times \mathbb{R}$ if $g_{n}(s, y, \theta)$ is modified only outside $\left(V_{0}^{\prime} \cap C_{-}\right) \times \mathbb{R}$.

Using these facts, one can easily check by induction on $k$ that $Z_{k}^{\#}, \partial_{\theta} Z_{k+1}$, $1 \leq k \leq \mu-1$, do not change in $\mathscr{D} \cap C_{-}$if $g^{i j}, g$ are replaced by $\hat{g}^{i j}, \hat{g}$ and if the initial data (4.14), (4.16), (4.34), (4.36) are kept unchanged. Hence $\widehat{Z}^{\varepsilon}=Z^{\varepsilon}$ in $\mathscr{D} \cap C_{-}$. Put $U^{\varepsilon}={ }^{\operatorname{tr}}\left(\hat{Z}^{\varepsilon}, D_{0} \widehat{Z}^{\varepsilon}, D_{1} \widehat{Z}^{\varepsilon}, \ldots, D_{N} \widehat{Z}^{\varepsilon}\right)$. It follows from the construction of $\widehat{Z}^{\varepsilon}$ that $U^{\varepsilon}(s, y)=0$ for all $(s, y) \in \mathscr{D}$ such that $|y| \geq s+r$. Let $\widehat{R}_{\mu}$ be the remainder corresponding to $R_{\mu}$ if $g^{i j}, g, Z^{\varepsilon}$ are replaced by $\hat{g}^{i j}, \hat{g}, \widehat{Z}^{\varepsilon}$. If

$$
R^{\varepsilon}(s, y)={ }^{\mathrm{tr}}\left(0, \widehat{R}_{\mu}\left(s, y, \frac{\Phi(s, y)}{\varepsilon}, \varepsilon\right), 0, \ldots, 0\right),
$$

we have

$$
D_{0} U^{\varepsilon}+\sum_{1 \leq j \leq N} A_{j}\left(s, y, U^{\varepsilon}\right) D_{j} U^{\varepsilon}-F\left(s, y, U^{\varepsilon}\right)=\varepsilon^{\mu} R^{\varepsilon}
$$

in $\mathscr{D}$. Let $L(y)$ be as in $\S 4$. To be able to apply immediately the results of [5], let us make the change of variables $(s, y) \mapsto(\tilde{s}, y)$, where $\tilde{s}=s / L(y)$. Assume that $\delta_{1}$ is so small that $I-\tilde{s} \sum_{1 \leq j \leq N} D_{j} L(y) \cdot A_{j}(L(y) \tilde{s}, y, \bar{U})$, where $I$ is the identity $(N+2) \times(N+2)$ matrix, is invertible if $-1 \leq \tilde{s} \leq 0, y \in \mathbb{R}^{N}$, and $\bar{U} \in \mathbb{R}^{N+2}$. Put

$$
\begin{aligned}
\widetilde{A}(\tilde{s}, y, \bar{U}) & =L(y)\left(I-\tilde{s} \sum_{1 \leq j \leq N} D_{j} L(y) \cdot A_{j}(L(y) \tilde{s}, y, \bar{U})\right)^{-1}, \\
\bar{A}_{j}(\tilde{s}, y, \bar{U}) & =\bar{A}(\tilde{s}, y, \bar{U}) A_{j}(L(y) \tilde{s}, y, \bar{U}), \\
\bar{F}(\tilde{s}, y, \bar{U}) & =\bar{A}(\tilde{s}, y, \bar{U}) F(L(y) \tilde{s}, y, \bar{U}), \\
\bar{U}^{\varepsilon}(\tilde{s}, y) & =U^{\varepsilon}(L(y) \tilde{s}, y), \\
\bar{R}^{\varepsilon}(\tilde{s}, y) & =\bar{A}\left(\tilde{s}, y, \bar{U}^{\varepsilon}(\tilde{s}, y)\right) R^{\varepsilon}(L(y) \tilde{s}, y) .
\end{aligned}
$$

(5.5) implies that $\partial_{\tilde{s}} \bar{U}^{\varepsilon}+\sum_{1 \leq j \leq N} \bar{A}_{j}\left(\tilde{s}, y, \bar{U}^{\varepsilon}\right) D_{j} \bar{U}^{\varepsilon}-\bar{F}\left(\tilde{s}, y, \bar{U}^{\varepsilon}\right)=\varepsilon^{\mu} \bar{R}^{\varepsilon}(\tilde{s}, y)$. To simplify the notations, let us write $s$ instead of $\tilde{s}$ from now on. Because 
of (5.5), we have in particular

$$
D_{0} \bar{U}^{\varepsilon}+\sum_{1 \leq j \leq N} \bar{A}_{j}\left(s, y, \bar{U}^{\varepsilon}\right) D_{j} \bar{U}^{\varepsilon}-\bar{F}\left(s, y, \bar{U}^{\varepsilon}\right)=\varepsilon^{\mu} \bar{R}^{\varepsilon}(s, y)
$$

if $-1<s<0, y \in \mathbb{R}^{N}$. From (4.41) and (4.10), it easily follows that the following holds: for each $\varepsilon_{0}>0$, one can find $C_{\alpha}>0$ such that

$$
\begin{aligned}
& \left|D^{\alpha} \bar{U}^{\varepsilon}(s, y)\right| \leq C_{\alpha} \varepsilon^{1-|\alpha|} \quad \text { and }\left|D^{\alpha} \bar{R}^{\varepsilon}(s, y)\right| \leq C_{\alpha} \varepsilon^{-|\alpha|} \\
& \qquad \text { if }-1 \leq s \leq 0, y \in \mathbb{R}^{N} \text {, and } 0<\varepsilon \leq \varepsilon_{0} .
\end{aligned}
$$

Furthermore,

$$
\bar{U}^{\varepsilon}(s, y) \text { and therefore } \bar{R}^{\varepsilon}(s, y) \text { vanish if }-1 \leq s \leq 0 \text { and }|y| \geq r .
$$

Now if we put $\bar{S}(s, y, \bar{U})=S(L(y) s, y, \bar{U})(\bar{A}(s, y, \bar{U}))^{-1}$, it is easily checked that

(5.4) holds with $S(s, y, U)$ replaced by $\bar{S}(s, y, \bar{U}), A_{j}(s, y, U)$ replaced by $\bar{A}_{j}(s, y, \bar{U}), \mathscr{B}$ replaced by $\{(s, y, \bar{U}) \in \mathbb{R} \times$ $\left.\mathbb{R}^{N} \times \mathbb{R}^{N+2},-1 \leq s \leq 0\right\}$, and $C_{1}, C_{2}$ replaced by new strictly positive constants $\bar{C}_{1}, \bar{C}_{2}$ respectively.

For a function $f(s, y)$ for which this makes sense, put

$$
\|f(s)\|=\left(\int_{\mathbb{R}^{N}}|f(s, y)|^{2} d y\right)^{1 / 2} .
$$

Choose $\mu>(N+3) / 2$. Put $r_{4}=\max \left(\left(9 r_{2}^{2}-1 / 4 t_{0}^{2}\right)^{1 / 2}, r_{3}\right)$. Let $v: \mathbb{R}^{N} \rightarrow \mathbb{R}^{N}$ be a $C^{\infty}$ function such that $v(y)=y$ if $|y| \leq r_{4},|v(y)| \geq r_{4}$ if $|y| \geq r_{4}$, and such that, for each $\alpha \in \mathbb{N}^{N}, \partial^{\alpha} v$ is uniformly bounded in $\mathbb{R}^{N}$. Put $\xi(s, y)=(s, v(y))$. Now if $-1 \leq s \leq 0,|y| \geq r_{4}$ and $\bar{U} \in \mathbb{R}^{N+2}$, then each $\bar{A}_{j}(s, y, \bar{U}), 1 \leq j \leq N$, is constant and $\bar{F}(s, y, \bar{U})$ is independent of $s$ and $y$. Therefore if $-1 \leq s \leq 0, y \in \mathbb{R}^{N}$, and $\bar{U} \in \mathbb{R}^{N+2}$, we have $\bar{A}_{j}(s, y, \bar{U})=$ $\bar{A}_{j}(\xi(s, y), \bar{U})$ for $1 \leq j \leq N$ and $\bar{F}(s, y, \bar{U})=\bar{F}(\xi(s, y), \bar{U})$. Since on the other hand (5.6), (5.7), (5.8), (5.9) hold, it follows from Theorem 1.1 of [5] that there exists $\varepsilon_{0}>0$ such that the following holds: if $0<\varepsilon \leq \varepsilon_{0}$, one can find $\bar{U}^{\ell} \in C^{\infty}\left([-1,0] \times \mathbb{R}^{N}\right)$ satisfying the following conditions:

$$
\begin{aligned}
D_{0} \underline{\bar{U}}^{\varepsilon}+\sum_{1 \leq j \leq N} \bar{A}_{j}\left(s, y, \underline{U}^{\varepsilon}\right) D_{j} \overline{\bar{U}}^{\varepsilon}-\bar{F}\left(s, y, \bar{U}^{\varepsilon}\right)=0 \\
\quad \text { if }-1<s<0, y \in \mathbb{R}^{N} ;
\end{aligned}
$$

$$
{\overline{U^{\varepsilon}}}^{\varepsilon}-\bar{U}^{\varepsilon}=0 \quad \text { if } s=-1, y \in \mathbb{R}^{N} ;
$$

for each $\alpha=\left(\alpha_{0}, \ldots, \alpha_{N}\right) \in \mathbb{N}^{N+1}$ such that $\alpha_{0}=0$ and $|\alpha| \leq$ $\mu$, one can find $C_{\alpha}>0$ such that $\left\|D^{\alpha}\left(\bar{U}^{\varepsilon}-\bar{U}^{\varepsilon}\right)(s)\right\| \leq C_{\alpha} \varepsilon^{\mu-|\alpha|}$ if $-1 \leq s \leq 0$ and $0<\varepsilon \leq \varepsilon_{0}$.

(In the present situation, the function $\bar{U}^{\varepsilon}$ provided by Theorem 1.1 of [5] is a $C^{\infty}$ function because its initial value $y \mapsto \underline{U}^{\varepsilon}(-1, y)$ is a $C_{0}^{\infty}$ function; this standard regularity property follows from [12] or [11].) The following proposition, which will be used later on, shows that we still have estimates of type (5.12) when we allow derivatives with respect to $s$. 
Proposition 5.1. (5.12) holds for all $\alpha \in \mathbb{N}^{N+1}$ such that $|\alpha| \leq \mu$.

We postpone the proof of Proposition 5.1 until the end of this section in order not to interrupt the proof of Theorem 2. If we now define $\underline{U}^{\varepsilon}(s, y)=$ $\bar{U}^{e}(s / L(y), y)$ for $(s, y) \in \mathscr{D}^{*}$, we obtain from (5.10) that

$$
D_{0} \underline{U}^{\varepsilon}+\sum_{1 \leq j \leq N} A_{j}\left(s, y, \underline{U}^{\varepsilon}\right) D_{j} \underline{U}^{\varepsilon}-F\left(s, y, \underline{U}^{\varepsilon}\right)=0 \quad \text { if }(s, y) \in \mathscr{D}^{*},
$$

and (5.11) gives that

$$
\underline{U}^{\varepsilon}-U^{\varepsilon}=0 \quad \text { if }(s, y) \in H^{*} .
$$

For $\lambda>0, \rho>(1+\lambda) r$, put $\mathscr{E}_{\lambda, \rho}=\left\{(s, y) \in \mathscr{D}^{*},(1+\lambda)(s+r)<|y|<\right.$ $\rho-(1+\lambda) s\}$. (The condition $\rho>(1+\lambda) r$ ensures that $\mathscr{E}_{\lambda, \rho} \neq \varnothing$.) Since (5.4) holds, we may apply the standard energy method (cf. e.g. [11]): we multiply (5.13) by $S\left(s, y, \underline{U}^{\varepsilon}\right)$, make the scalar product with $\underline{U}^{\varepsilon}$ and integrate by parts over $\mathscr{E}_{\lambda, \rho}$, using (5.14). The conclusion is that one can find $\lambda_{0}>0$ such that $\underline{U}^{\varepsilon}=0$ on $\mathscr{E}_{\lambda, \rho}$ if $\lambda \geq \lambda_{0}$ and $\rho>(1+\lambda) r$. Letting $\rho$ tend to $+\infty$, we obtain that $\lambda_{0} \in \mathscr{H}$ if $\mathscr{H}=\left\{\lambda \geq 0, \underline{U}^{\varepsilon}(s, y)=0\right.$ if $(s, y) \in \mathscr{D}^{*}$ and $|y| \geq(1+\lambda)(s+r)\}$. Now $\mathscr{H}$ is a half-line; $\mathscr{H}$ is closed in $[0,+\infty[$ and the energy method also shows that $\mathscr{H}$ is open in $[0,+\infty[$. Therefore $0 \in \mathscr{H}$ and so $\underline{U}^{\varepsilon}(s, y)=0$ if $|y| \geq s+r$.

Denote by $\left(\underline{U}_{j}^{\varepsilon}\right), 1 \leq j \leq N+2$, the components of $\underline{U}^{\varepsilon}$, so that $\underline{U}^{\varepsilon}=$ ${ }^{\text {ti }}\left(\underline{U}_{1}^{\varepsilon}, \ldots, \underline{U}_{N+2}^{\varepsilon}\right)$. From (5.13) it follows that, on $\mathscr{D}^{*}$, we have $D_{0} \underline{U}_{1}^{\varepsilon}=\underline{U}_{2}^{\varepsilon}$ and $D_{0}\left(\underline{U}_{2+j}^{\varepsilon}-D_{j} \underline{U}_{1}^{\varepsilon}\right)=0$ if $1 \leq j \leq N$. On the other hand (5.14) and the definition of $U^{\varepsilon}$ show that $\underline{U}_{2+j}^{\varepsilon}=D_{j} \underline{U}_{1}^{\varepsilon}$ on $H^{*}$ if $1 \leq j \leq N$. Therefore $\underline{U}_{2+j}^{\varepsilon}=D_{j} \underline{U}_{1}^{\varepsilon}$ on $\mathscr{D}^{*}$ if $0 \leq j \leq N$. Hence if we put $\underline{Z}^{\varepsilon}=\underline{U}_{1}^{\varepsilon}$, it follows from (5.13) that $\underline{Z}^{\varepsilon}$ is a solution of (5.1) in $\mathscr{D}^{*}$, whereas (5.14) implies that $\underline{Z}^{\varepsilon}=Z^{\varepsilon}$ on $H^{*}$. Now put $\underline{Z}^{\varepsilon}(s, y)=0$ if $(s, y) \in \mathscr{D} \backslash \mathscr{D}^{*}$. Then $\underline{Z}^{\varepsilon} \in C^{\infty}(\overline{\mathscr{D}}), \underline{Z}^{\varepsilon}$ is a solution of (5.1) in $\mathscr{D}$, and $\underline{Z}^{\varepsilon}=Z^{\varepsilon}$ on $H$. If $-1 \leq s \leq 0$ and $y \in \mathbb{R}^{N}$, put $\underline{\bar{Z}}^{\varepsilon}(s, y)=\underline{Z}^{\varepsilon}(L(y) s, y), \bar{Z}^{\varepsilon}(s, y)=\widehat{Z}^{\varepsilon}(L(y) s, y)$, so that $\underline{\bar{Z}}^{\varepsilon}$ and $\bar{Z}^{\varepsilon}$ are the first components of $\bar{U}^{\varepsilon}$ and $\bar{U}^{\varepsilon}$ respectively. Using Proposition 5.1 and applying Sobolev's imbedding theorem in the $y$ variables, we obtain that one can find $C_{\alpha}>0$ such that

$$
\begin{aligned}
\left|D^{\alpha+\nu}\left(\underline{\bar{Z}}^{\varepsilon}-\bar{Z}^{\varepsilon}\right)(s, y)\right| \leq C_{\alpha} \varepsilon^{\mu-(N+1) / 2-|\alpha|} & \text { if }-1 \leq s \leq 0, y \in \mathbb{R}^{N}, 0<\varepsilon \leq \varepsilon_{0}, \\
& |\nu| \leq 1, \text { and }|\alpha| \leq \mu-(N+1) / 2 .
\end{aligned}
$$

From (5.15) it follows immediately that, for some $C_{\alpha}^{\prime}>0$,

$$
\begin{aligned}
& \left|D^{\alpha+\nu}\left(\underline{Z}^{\varepsilon}-\widehat{Z}^{\varepsilon}\right)(s, y)\right| \leq C_{\alpha}^{\prime} \varepsilon^{\mu-(N+1) / 2-|\alpha|} \\
& \quad \text { if }(s, y) \in \mathscr{D}, 0<\varepsilon \leq \varepsilon_{0},|\nu| \leq 1 \text {, and }|\alpha| \leq \mu-(N+1) / 2 .
\end{aligned}
$$

From Proposition 5.1 it follows at once that, for some $C_{\alpha}^{\prime \prime}>0$,

$$
\begin{aligned}
\iint_{\mathscr{D}}\left|D^{\alpha+\nu}\left(\underline{Z}^{\varepsilon}-\hat{Z}^{\varepsilon}\right)(s, y)\right|^{2} d s d y & \leq C_{\alpha}^{\prime \prime} \varepsilon^{2(\mu-|\alpha|)} \\
& \text { if } 0<\varepsilon \leq \varepsilon_{0},|\nu| \leq 1, \text { and }|\alpha| \leq \mu .
\end{aligned}
$$

We may now complete the proof of Theorem 2. Assume first that $t_{0}>0$ is so large that all the steps of the construction of $Z^{\varepsilon}$ and all the estimates of 
$Z^{\varepsilon}$ described in $\S 4$ are valid. As already pointed out before (5.5), we have $Z^{\varepsilon}=\widehat{Z}^{\varepsilon}$ in $\mathscr{D} \cap C_{-}$. Furthermore, if $\varepsilon$ is small enough, it follows from (5.16), (4.41), and the definition of $\hat{g}^{i j}, \hat{g}$ that $\underline{Z}^{\varepsilon}$ is a solution of (3.1) in $\mathscr{D} \cap C_{-}$. Let $z^{\varepsilon}$ be as defined at the end of $\S 4$; recall that $z^{\varepsilon}(t, x)=0$ if $t \geq t_{0}$ and $|x| \geq t+M-t_{0}$. Now put $\underline{z}^{\varepsilon}(t, x)=\left(t^{2}-|x|^{2}\right)^{-(N-1) / 2} \underline{Z}^{\varepsilon}(J(t, x))$ if $t \geq t_{0}$ and $(t, x) \in C_{+}, \underline{z}^{\varepsilon}(t, x)=0$ if $t \geq t_{0}$ and $(t, x) \notin C_{+}$. Then $\square \underline{z}^{\varepsilon}-\sum_{0 \leq i, j \leq N} f^{i j}\left(\underline{z}^{\varepsilon},\left(\underline{z}^{\varepsilon}\right)^{\prime}\right) \partial_{i j}^{2} \underline{z}^{\varepsilon}-f\left(\underline{z}^{\varepsilon},\left(\underline{z}^{\varepsilon}\right)^{\prime}\right)=0$ if $t>t_{0}$ and $(t, x) \in C_{+}$, and $\partial_{0}^{k}\left(\underline{z}^{\varepsilon}-z^{\varepsilon}\right)\left(t_{0}, x\right)=0$ if $k=0,1$ and $x \in \mathbb{R}^{N}$. As noticed after (5.14), $\underline{U}^{\varepsilon}(s, y)=0$ if $(s, y) \in \mathscr{D}^{*}$ and $|y| \geq s+r$. Hence $\underline{Z}^{\varepsilon}(s, y)=0$ if $(s, y) \in \mathscr{D}$ and $|y| \geq s+r$, and since $r=\left(t_{0}-M\right)^{-1}$, it follows that $\underline{z}^{\varepsilon}(t, x)=0$ if $t \geq t_{0}$, $|x| \geq t+M-t_{0}$, and $(t, x) \in C_{+}$. Hence $\underline{z}^{\varepsilon} \in C^{\infty}\left(\left[t_{0},+\infty\left[\times \mathbb{R}^{N}\right)\right.\right.$ and $\underline{z}^{\varepsilon}$ is a solution of $(2.1)$. Notice that

$$
\left(z^{\varepsilon}-\underline{z}^{\varepsilon}\right)(t, x)=0 \quad \text { if } t \geq t_{0} \text { and }|x| \geq t+M-t_{0} .
$$

Part (i) of Theorem 2 follows at once from (5.15) and (5.18). Now

$$
\begin{aligned}
& \text { the Jacobian determinant of } J \text { at }(t, x) \text { is equal to } \\
& (-1)^{N}\left(t^{2}-|x|^{2}\right)^{-(N+1)} \text {. }
\end{aligned}
$$

Actually since $J$ commutes with the orthogonal transformations in the $x$ variables, it is enough to prove (5.19) when $x_{j}=0$ for $j \geq 2$, in which case it follows easily by induction on $N$. Part (ii) of Theorem 2 follows easily from (5.17), (5.19). This completes the proof of Theorem 2 in case $t_{0}$ is large enough. Now if $t_{0}$ is any real number, Theorem 2 also follows because we may, by a translation of $t$, reduce to the case where $t_{0}>0$ is as large as we please. The proof of Theorem 2 is complete.

It only remains to prove Proposition 5.1.

Proof of Proposition 5.1. Put $W^{\varepsilon}=\bar{U}^{\varepsilon}-\bar{U}^{\ell}$. Subtracting (5.6) from (5.10) and making use of the function $\xi$ introduced just before (5.10), we obtain

$$
\begin{aligned}
D_{0} W^{\varepsilon} & +\sum_{1 \leq j \leq N} \bar{A}_{j}\left(\xi, \bar{U}^{\varepsilon}\right) D_{j} W^{\varepsilon}+S_{1}\left(\xi, \bar{U}^{\varepsilon}, \bar{U}^{\varepsilon}, D_{y} \bar{U}^{\varepsilon}\right) W^{\varepsilon} \\
& =S_{2}\left(\xi, \underline{U}^{\varepsilon}, \bar{U}^{\varepsilon}\right) W^{\varepsilon}-\varepsilon^{\mu} \bar{R}^{\varepsilon}
\end{aligned}
$$

if $-1<s<0$ and $y \in \mathbb{R}^{N}$. Here $S_{1}, S_{2}$ are $(N+2) \times(N+2)$ matrices depending in a $C^{\infty}$ way on their arguments, and $S_{1}\left(\xi, \underline{U}^{\varepsilon}, \bar{U}^{\varepsilon}, D_{y} \bar{U}^{\varepsilon}\right)$ is a linear function of $D_{k} \bar{U}_{l}^{\varepsilon}, 1 \leq k \leq N, 1 \leq l \leq N+2$, where of course $\bar{U}_{l}^{\varepsilon}$ is the $l$ th component of $\bar{U}^{\varepsilon}$. Now put $w^{\varepsilon}(s, y)=W^{\varepsilon}(\varepsilon s, \varepsilon y), \bar{u}^{\varepsilon}(s, y)=$ $\bar{U}^{\varepsilon}(\varepsilon s, \varepsilon y), \quad \xi^{\varepsilon}(s, y)=\xi(\varepsilon s, \varepsilon y), \quad Y^{\varepsilon}(s, y)=\varepsilon^{\mu+1} \bar{R}^{\varepsilon}(\varepsilon s, \varepsilon y)$. Then (5.20) gives

$$
\begin{aligned}
D_{0} w^{\varepsilon}= & -\sum_{1 \leq j \leq N} \bar{A}_{j}\left(\xi^{\varepsilon}, \bar{u}^{\varepsilon}+w^{\varepsilon}\right) D_{j} w^{\varepsilon} \\
& -S_{1}\left(\xi^{\varepsilon}, \bar{u}^{\varepsilon}+w^{\varepsilon}, \bar{u}^{\varepsilon}, D_{y} \bar{u}^{\varepsilon}\right) w^{\varepsilon}+\varepsilon S_{2}\left(\xi^{\varepsilon}, \bar{u}^{\varepsilon}+w^{\varepsilon}, \bar{u}^{\varepsilon}\right) w^{\varepsilon}-Y^{\varepsilon},
\end{aligned}
$$

when $-1 / \varepsilon<s<0$ and $y \in \mathbb{R}^{N}$. Now one can find constants $\varepsilon_{0}>0, C_{\alpha}>0$, 
$C>0$, such that the following holds for all $\left.\varepsilon \in] 0, \varepsilon_{0}\right]$ :

$$
\left|D^{\alpha} \xi^{\varepsilon}(s, y)\right| \leq C_{\alpha} \varepsilon^{|\alpha|} \quad \text { if } \alpha \in \mathbb{N}^{N+1},-\frac{1}{\varepsilon} \leq s \leq 0 \text { and } y \in \mathbb{R}^{N} ;
$$

$$
\left|D^{\alpha} \bar{u}^{\varepsilon}(s, y)\right| \leq C_{\alpha} \varepsilon \quad \text { if } \alpha \in \mathbb{N}^{N+1},-\frac{1}{\varepsilon} \leq s \leq 0 \text { and } y \in \mathbb{R}^{N} ;
$$

$\left|D^{\alpha} Y^{\varepsilon}(s, y)\right| \leq C_{\alpha} \varepsilon^{\mu+1} \quad$ if $\alpha \in \mathbb{N}^{N+1},-\frac{1}{\varepsilon} \leq s \leq 0$ and $y \in \mathbb{R}^{N}$

$$
\left\|D^{\alpha} Y^{\varepsilon}(s)\right\| \leq C_{\alpha} \varepsilon^{\mu+1-N / 2} \quad \text { if } \alpha \in \mathbb{N}^{N+1} \text { and }-\frac{1}{\varepsilon} \leq s \leq 0 ;
$$

$\left\|D^{\alpha} w^{\varepsilon}(s)\right\| \leq C_{\alpha} \varepsilon^{\mu-N / 2} \quad$ if $\alpha \in \mathbb{N}^{N+1}, \alpha_{0}=0,|\alpha| \leq \mu$, and $-\frac{1}{\varepsilon} \leq s \leq 0 ;$

$$
\left|w^{\varepsilon}(s, y)\right| \leq C \varepsilon^{\mu-N / 2} \quad \text { if }-\frac{1}{\varepsilon} \leq s \leq 0 \text { and } y \in \mathbb{R}^{N} .
$$

Actually (5.22) is straightforward and (5.23), (5.24) follow at once from (5.7). Since $\bar{U}^{\varepsilon}(s, y)=0$ for $|y| \geq r$, we also have $\bar{R}^{\varepsilon}(s, y)=0$ for $|y| \geq r$, so that (5.25) follows from (5.24). (5.26) is a consequence of (5.12) and (5.27) follows from (5.26) and Sobolev's imebdding theorem. To prove Proposition 5.1 , it is enough to show that (5.26) still holds if $\alpha_{0} \neq 0$ and $|\alpha| \leq \mu$. Let us show this. To shorten the notation, if $n \in \mathbb{N}$, put $\bar{D}^{n} f=\left\{D^{\beta} f,|\beta| \leq n\right\}$. For $1 \leq k \leq N+2$, denote by $D^{\beta} w_{k}^{\varepsilon}$ the component number $k$ of $D^{\beta} w^{\varepsilon}$ and by $D^{\beta} Y_{k}^{\varepsilon}$ the component number $k$ of $D^{\beta} Y^{\varepsilon}$. If $\alpha_{0} \neq 0$ and $1 \leq k \leq N+2$, we may write

$$
D^{\alpha} w_{k}^{\varepsilon}=\mathscr{S}_{\alpha k}^{\varepsilon}+\mathscr{T}_{\alpha k}^{\varepsilon}+\mathscr{U}_{\alpha k}^{\varepsilon} .
$$

In (5.28), $\mathscr{S}_{\alpha k}^{\varepsilon}$ is a sum of terms of the form

$$
\varepsilon^{i} G\left(\bar{D}^{j} \xi^{\varepsilon}, \bar{D}^{n} \bar{u}^{\varepsilon}, \bar{D}^{q} Y^{\varepsilon}, w^{\varepsilon}\right) \prod_{\sigma, l}\left(D_{y}^{\sigma} w_{l}^{\varepsilon}\right)^{\rho \sigma t},
$$

where $i \in \mathbb{N}, i \leq|\alpha|-1, G \in C^{\infty}$, and $\sigma \neq 0, \rho_{\sigma l} \neq 0, j+n+q+$ $\sum_{\sigma, l}|\sigma| \rho_{\sigma l} \leq|\alpha| ; \mathscr{T}_{\alpha k}^{\varepsilon}$ is a sum of terms of the form

$$
\varepsilon^{i} H\left(\bar{D}^{j} \xi^{\varepsilon}, \bar{D}^{n} \bar{u}^{\varepsilon}, \bar{D}^{q} Y^{\varepsilon}, w^{\varepsilon}\right) w_{l}^{\varepsilon}
$$

where $i \in \mathbb{N}, i \leq|\alpha|, H \in C^{\infty}, j+n+q \leq|\alpha| ; \mathscr{U}_{\alpha k}^{\varepsilon}$ is a sum of terms of the form

$$
\varepsilon^{i} K\left(\bar{D}^{j} \xi^{\varepsilon}, \bar{D}^{n} \bar{u}^{\varepsilon}, \bar{D}^{q} Y^{\varepsilon}, w^{\varepsilon}\right) D^{\gamma} Y_{l}^{\varepsilon},
$$

where $i \in \mathbb{N}, i \leq|\alpha|-1, K \in C^{\infty}, j+n+q+|\gamma| \leq|\alpha|-1$. In fact (5.21) shows that (5.28) holds if $|\alpha|=1$, and the general case is easily obtained by induction. Now $\mathscr{S}_{\alpha k}^{\varepsilon}$ can be estimated by using the same arguments as in the proof of Moser's inequality (see e.g. the proof of Lemma 5.1 of [7]). To bound a norm $\left\|\prod_{\sigma_{l}(}\left(D_{y}^{\sigma} w_{l}^{\varepsilon}\right)^{\rho_{\sigma l}}(s)\right\|$ where $-\frac{1}{\varepsilon} \leq s \leq 0$, put $\nu=\sum_{\sigma, l}|\sigma| \rho_{\sigma l}$ (where the sum is extended over all $\sigma, l$ occurring in the product), $p_{\sigma l}=2 \nu /|\sigma| \rho_{\sigma l}$. For a function $f(s, y)$ for which this makes sense, write $\|f(s)\|_{L^{p}\left(\mathbb{R}_{y}^{N}\right)}=\left(\int_{\mathbb{R}^{N}}|f(s, y)|^{p} d y\right)^{1 / p}$ if $1 \leq p<+\infty$. Then Hölder's 
inequality gives

$$
\begin{aligned}
\left\|\prod_{\sigma, l}\left(D_{y}^{\sigma} w_{l}^{\varepsilon}\right)^{\rho_{\sigma l}}(s)\right\| & \leq \prod_{\sigma, l}\left\|\left(D_{y}^{\sigma} w_{l}^{\varepsilon}\right)^{\rho_{\sigma l}}(s)\right\|_{L^{p_{\sigma l}\left(\mathbb{R}_{y}^{N}\right)}} \\
& =\prod_{\sigma, l}\left\|\left(D_{y}^{\sigma} w_{l}^{\varepsilon}\right)(s)\right\|_{L^{2 \nu /|\sigma|}\left(\mathbb{R}_{y}^{N}\right)}^{\rho_{\sigma l}}
\end{aligned}
$$

By the Gagliardo-Nirenberg estimates we have

$$
\left\|\left(D_{y}^{\sigma} w_{l}^{\varepsilon}\right)(s)\right\|_{L^{2 \nu /|\sigma|}\left(\mathbb{R}_{y}^{N}\right)} \leq C_{\sigma}\left(\sup _{y \in \mathbb{R}^{n}}\left|w^{\varepsilon}(s, y)\right|\right)^{1-|\sigma| / \nu} \cdot \sum_{|\gamma|=\nu}\left\|D_{y}^{\gamma} w^{\varepsilon}(s)\right\|^{|\sigma| / \nu} ;
$$

using (5.27) and (5.26), we therefore obtain, since $\nu \leq|\alpha| \leq \mu$ :

(5.30) $\left\|\left(D_{y}^{\sigma} w_{l}^{\varepsilon}\right)(s)\right\|_{L^{2 \nu /|\sigma|}\left(\mathbb{R}_{y}^{N}\right)} \leq C_{\sigma} \varepsilon^{\mu-N / 2} \quad$ if $-\frac{1}{\varepsilon} \leq s \leq 0$ and $0<\varepsilon \leq \varepsilon_{0}$.

But then (5.22), (5.23), (5.24), (5.27), (5.29), (5.30) show that

$$
\left\|\mathscr{S}_{\alpha k}^{\varepsilon}(s)\right\| \leq C_{\alpha} \varepsilon^{\mu-N / 2} \quad \text { if }-\frac{1}{\varepsilon} \leq s \leq 0 \text { and } 0<\varepsilon \leq \varepsilon_{0} .
$$

On the other hand, because of (5.22), (5.23), (5.24), (5.27), we have $\left\|\mathscr{T}_{\alpha k}^{\varepsilon}(s)\right\|$ $\leq C\left\|w^{\varepsilon}(s)\right\|$ if $-1 / \varepsilon \leq s \leq 0$ and $0<\varepsilon \leq \varepsilon_{0}$. Hence (5.26) implies that

$$
\left\|\mathscr{T}_{\alpha k}^{\varepsilon}(s)\right\| \leq C_{\alpha} \varepsilon^{\mu-N / 2} \quad \text { if }-\frac{1}{\varepsilon} \leq s \leq 0 \text { and } 0<\varepsilon \leq \varepsilon_{0} .
$$

Finally (5.22), (5.23), (5.24), (5.27) show that

$$
\left\|\mathscr{U}_{\alpha k}^{\varepsilon}(s)\right\| \leq C_{\alpha} \sum_{|\gamma| \leq|\alpha|-1}\left\|D^{\gamma} Y^{\varepsilon}(s)\right\|
$$

if $-1 / \varepsilon \leq s \leq 0$ and $0<\varepsilon \leq \varepsilon_{0}$. By (5.25) it follows that

$$
\left\|\mathscr{U}_{\alpha k}^{\varepsilon}(s)\right\| \leq C_{\alpha} \varepsilon^{\mu+1-N / 2} \quad \text { if }-\frac{1}{\varepsilon} \leq s \leq 0 \text { and } 0<\varepsilon \leq \varepsilon_{0} .
$$

From (5.28), (5.31), (5.32), (5.33), it follows that (5.26) still holds if $\alpha_{0} \neq 0$ and $|\alpha| \leq \mu$. This completes the proof of Proposition 5.1.

\section{REFERENCES}

1. D. Christodoulou, Global solutions of nonlinear hyperbolic equations for small initial data, Comm. Pure Appl. Math. 39 (1986), 267-282.

2. R. Courant and D. Hilbert, Methods of mathematical physics, Vol. 2, Interscience, 1962.

3. P. Godin, Long time existence of piecewise smooth progressing waves for semilinear wave equations, J. Math. Pures Appl. 72 (1993), 15-56.

4. _ Global sound waves for quasilinear second order wave equations, Math. Ann. 298 (1994), 497-531.

5. O. Guès, Développement asymptotique de solutions exactes de systèmes hyperboliques quasilinéaires, Asymptotic Analysis 6 (1993), 241-269.

6. J. L. Joly, G. Métivier, and J. Rauch, Remarques sur l'optique géométrique non linéaire multidimensionnelle, exposé no. 1, Séminaire EDP, Ecole Polytechnique, Palaiseau, 1990-91.

7. S. Klainerman, Global existence for nonlinear wave equations, Comm. Pure Appl. Math. 33 (1980), 43-101. 
8. Uniform decay estimates and the Lorentz invariance of the classical wave equation, Comm. Pure Appl. Math. 38 (1985), 321-332.

9. Appl. Math., vol. 23, Amer. Math. Soc., Providence, RI, 1986, pp. 293-326.

10. T. T. Li and Y. M. Chen, Initial value problems for nonlinear wave equations, Comm. Partial Differential Equations 13 (1988), 383-422.

11. A. Majda, Compressible fluid flow and systems of conservation laws in several space variables, Appl. Math. Sci., vol. 53, Springer, 1984.

12. G. Métivier, Problèmes de Cauchy et ondes non linéaires, Actes du Colloque de Saint-Jeande-Monts 1986, exposé no. 1, Editions de l'Ecole Polytechnique, Palaiseau, 1986.

13. K. Morawetz, Energy decay for star-shaped obstacles, in Scattering Theory (P. D. Lax and R. Phillips), Academic Press, 1967, pp. 261-264.

14. M. Spivak, A comprehensive introduction to differential geometry, Vols. 3, 4, Publish or Perish, 1979.

Département de Mathématique, Université Libre de Bruxelles, Campus Plaine CP 214, BouleVARd DU TRIOMPHE, 1050 BRUXelles, BelgiUm

E-mail address: ulbmath@ulb.ac.be 